\title{
ON THE EXISTENCE OF MAASS CUSP FORMS ON HYPERBOLIC SURFACES WITH CONE POINTS
}

\author{
CHRISTOPHER M. JUDGE
}

\section{INTRODUCTION}

1.1. The Roelcke-Selberg Problem. To each finite volume hyperbolic surface $(M, g)$, one can associate a Laplace-Beltrami operator $\Delta: L^{2}\left(M, d V_{g}\right) \rightarrow$ $L^{2}\left(M, d V_{g}\right)$ where $d V_{g}$ is the $g$-volume form. Specifically, $\Delta$ is defined for a distribution $v \in H^{2}\left(M, d V_{g}\right)$ to be

$$
(\Delta v)(\phi)=-\int_{M} \operatorname{div}(\operatorname{grad}(\phi)) \cdot v d V_{g}
$$

where $\phi$ is a bounded $C^{\infty}(M)$ function. If the surface has $k$ cusps, then $\Delta$ has a continuous band of spectrum $\left[\frac{1}{4}, \infty\right)$ with multiplicity $k$. Aside from the continuous spectrum and finitely many eigenvalues in the interval $\left[0, \frac{1}{4}\right)$, the Laplace-Beltrami operator can have eigenvalues embedded in its continuous spectrum. The eigenfunctions associated to these embedded eigenvalues are called (Maass) cusp forms.

Using the trace formula, A. Selberg showed that the upper half plane quotients of congruence subgroups of $S L(2, \mathbf{Z})$ have infinitely many cusp forms. In fact, they have enough eigenvalues to account for the analogue of Weyl's Law. Selberg, and independently Roelcke, conjectured that for every co-finite discrete subgroup $\Gamma$ of $S L(2, \mathbf{R})$, the corresponding upper half plane quotient $\mathbf{H}^{2} / \Gamma$ has infinitely many cusp forms associated to its Laplace-Beltrami operator. This has come to be known as the Roelcke-Selberg Conjecture [T], [V].

However, based on the evidence collected to date, this conjecture appears to be false [CdV], [PS1], [PS2], [DIPS], [Wk], [H2], [Lu] [Wp2], [PS3]. This opinion is expressed in two types of conjecture: R. Phillips and P. Sarnak have conjectured that for $\chi(M)<0$ the generic surface has only finitely many cusp forms [DIPS], [PS2]; and Sarnak has proposed that a non-cocompact lattice in

Received by the editors August 2, 1993 and, in revised form, May 6, 1994.

1991 Mathematics Subject Classification. Primary 11F72; Secondary 58G25.

Key words and phrases. Maass cusp form, embedded eigenvalues, Hecke triangle group, hyperbolic surfaces.

Partially supported by NSF grant DMS 9304580. 
$S L(2, \mathbf{R})$ has an "essentially cuspidal" quotient if and only if the lattice is arithmetic [Sa2], [GGP]. ${ }^{1}$ Note that the set of arithmetic lattice quotients is a finite subset of the moduli space of complete, finite volume, hyperbolic structures on a differentiable surface. Yet, to date no one has found a single example of a non-cocompact lattice quotient for which the corresponding Laplace-Beltrami operator has spectral asymptotics differing from those which had been expected by Selberg.

The genesis of the recent work on the Roelcke-Selberg problem is a paper of Y. Colin de Verdiere [CdV]. Colin de Verdiere considers the moduli space of surfaces of variable curvature having finitely many hyperbolic cusps [CdV]. By considering compact deformations of the metric within a fixed conformal class and by using an operator introduced by Lax and Phillips [LP], the pseudo-Laplacian, he shows that the generic surface in this moduli space has no embedded eigenvalues [CdV]. Note, however, that the complete, finite volume, hyperbolic metrics constitute a finite-dimensional submanifold within the infinite-dimensional space considered in [CdV].

In the present paper conformal deformations are again considered. However, here the deformations strictly fall within the finite-dimensional Teichmüller space of conically singular hyperbolic metrics. The geometric deformation theory of such metrics is discussed in [J]. Let $\mathscr{T}_{g, n, m}$ be the Teichmüller space of hyperbolic metrics on a genus $g$ surface with $n$ conical singularities and $m$ cusps. (See Definition 2.1.) In the present paper, the following is proven:

Theorem 1.1 (Theorem 6.1). If there exists a metric in $\mathscr{T}_{g, n-1, m}(m>0)$ whose Laplacian has only simple embedded eigenvalues, then the Laplacian of the generic point in $\mathscr{T}_{g, n, m}$ has no Maass cusp forms. In particular the Laplacian of the generic point has only finitely many $L^{2}$ eigenfunctions.

A generic point is defined to be a point in a residual set.

It must be emphasized that the conclusion of Theorem 6.1 does not contradict the Roelcke-Selberg Conjecture nor affirm the Phillips-Sarnak Conjecture: The generic conically singular hyperbolic metric is not Riemannian complete nor obtained as a quotient of the upper half plane through the action of a discrete subgroup of $\operatorname{PSL}(2, \mathbf{R})$. Moreover, validating the simplicity hypothesis of Theorem 6.1 is non-trivial. In fact, multiplicity of eigenvalues is the main obstacle to all further progress on the conjecture. (Compare with [Lu], [Wp2], [PS3], and [JZ].) In the case considered in [CdV], generic simplicity followed from a soft result in [U]. But here the space of metrics considered is finite dimensional, and hence generic simplicity is a much more subtle issue.

1.2. The Hecke triangle deformation. The quotients of the Hecke triangle groups $G_{k}$ are examples of conically singular hyperbolic metrics which are not Riemannian complete. D. Hejhal has conducted an extensive numerical study of the eigenvalues of some of these groups with intriguing results [H2]. First

\footnotetext{
${ }^{1}$ Contrast with [Ef].
} 
note that $\mathbf{H}^{2} / G_{k}$ has an involutive isometry which decomposes $L^{2}\left(\mathbf{H}^{2} / G_{k}\right)$ into 'even' and 'odd' functions. Hejhal's computer software finds that the 'odd' eigenfunctions are plentiful. This is as expected because the generalized eigenfunctions which span the continuous spectrum are known to be 'even'. However, not a single 'even' eigenfunction for the non-arithmetic Hecke groups has been found. In contrast, Hejhal's computer program finds plenty of 'even' eigenfunctions associated to arithmetic $G_{k}(k=3,4,6)$. Note that Winkler has obtained similar results [Wk].

The Hecke triangle groups belong to a real-analytic one-parameter metric deformation which is described in detail in [J]. By applying the analogue of the method developed in [PS1], [Wp1], and [Wp2], it is shown below that the generic metric in this deformation has no associated 'even' eigenvalues-embedded or otherwise-assuming that the multiplicities of the eigenvalues associated to $\Gamma_{0}(2)$ and $S L(2, \mathbf{Z})$ are as expected. In particular, one has

Theorem 1.2 (Theorem 6.2). Assuming Hypothesis 6.1, at most a countable number of metrics in the Hecke triangle deformation $H(t)$ have even cusp forms associated to them.

Using this method, Wolpert had found similar results for hyperbolic surfaces with cusps and reflection symmetries [Wp2]. More recently R. S. Phillips and P. Sarnak have applied this method to the character case with significant success [PS3].

As a corollary we have the following answer to the motivational question of [PS4]:

Corollary 1.1 (Corollary 6.2). Assuming Hypothesis 6.1, the generic hyperbolic triangle with exactly one null angle has no associated Neumann eigenvalues.

As in [PS3], one can weaken the multiplicity Hypothesis 6.1 and obtain a conclusion which contradicts the stronger analogue of Selberg's original conjecture: "Each $(M, g)$ is essentially cuspidal " [Sa2], [PS3]. The statement of this result is given as Theorem 6.3.

1.3. Monotonicity and bounds on eigenvalues. Of perhaps independent interest is the monotone behavior of eigenvalue branches as cone angles are varied in a uniform direction (Theorem 5.1). This monotonicity of eigenvalues yields the following corollaries:

Corollary 1.2 (Corollary 5.1). Let $T$ and $T^{\prime}$ be geodesic triangles in $\mathbf{H}^{2}$ with cone angle triples $\left(\alpha_{1}, \alpha_{2}, \alpha_{3}\right)$ and $\left(\beta_{1}, \beta_{2}, \beta_{3}\right)$ such that $\alpha_{i}>\beta_{i}>0$ for $i=1,2$, and 3. Arrange the respective eigenvalues (including multiplicities) of the Neumann problem in increasing order. Then the $k^{\text {th }}$ Neumann eigenvalue of $T$ is greater than the $k^{\text {th }}$ Neumann eigenvalue of $T^{\prime}$.

Corollary 1.3 (Corollary 5.2). The odd-eigenvalues associated to the Hecke triangle groups are uniformly bounded from below by $\frac{1}{2} \pi^{2}$. 
Monotonicity is also used in a crucial way in the proof of Theorems 6.1 and 6.2 .

1.4. Survey of contents. The geometric prerequisites for the present study are contained in $[\mathrm{J}]$. However, to make the present exposition relatively self-contained, the relevant results and notational conventions contained in $[\mathrm{J}]$ are given in Section 2.

The bulk of this paper consists of studying the variational behavior of the spectrum of the Laplacian under metric perturbation. The emphasis is placed on developing the analytical tools needed to prove the disappearance theorems (Theorems 6.2 and 6.1). Most of the analysis concerns the sidestepping of the continuous spectrum whose existence makes a direct perturbational study of eigenvalues formidable. The main analytical tool is a pseudo-Laplacian generalized in the context of conically singular hyperbolic metrics. In Section 3, this generalization, dubbed the conical pseudo-Laplacian, is defined and its basic properties are delineated. In the latter half of Section 3, this operator is used to examine the limiting behavior of eigenfunctions of the usual Laplacian as the angle of a cone tends to some limit. In particular, it is shown that as the cone angle tends to zero, embedded eigenvalues tend to an embedded eigenvalue associated to the limiting metric (Proposition 3.3).

In Section 4, the existence and regularity of the eigenbranches of the conical pseudo-Laplacian are considered. It is found that if the angles do not vary through 0 , then the pseudo-Laplacians are analytic in the sense of Kato for real-analytic perturbations of the metric. In particular, the eigenvalues depend analytically on the angle parameter and the eigenfunctions vary real-analytically in $L^{2}$. However, if the angles vary through 0 , then the singular nature of the spectral perturbation requires that asymptotic perturbation theory be applied. In particular, asymptotic perturbation theory is used to show the existence of differentiable eigenbranches and is used to determine the behavior of the first variational term of an eigenfunction branch.

In Section 5, the monotonicity of eigenvalue branches of the conical pseudoLaplacian is demonstrated $(\kappa \equiv-1)$. Proofs for Corollaries 5.1 and 5.2 are also provided. In Section 6 , the results of the previous sections are combined to provide proofs of Theorems 6.2 and 6.1.

All of the 'hard' analysis has been deposited in Appendix A. In particular, the Fourier expansion of an eigenfunction in the model hyperbolic cone is considered there. The differential equations satisfied by the Fourier coefficients are examined with particular attention paid to the asymptotics of the solutions near the cone point.

\section{GEOMETRIC PRELIMINARIES}

This section is a summary of the spectrally relevant results and notational conventions found in [J]. Details can be found there. 
The model hyperbolic cone is defined to be the metric

$$
\frac{\alpha^{2}\left(d x^{2}+d y^{2}\right)}{(\sinh \alpha y)^{2}}
$$

on the cylinder $S^{1} \times \mathbf{R}^{+}$. The cone point is the ideal point associated to $S^{1} \times$ $\{\infty\}$. The real constant $\alpha$ will denote the cone angle. Note that by letting $\alpha$ tend to zero in (2), one obtains

$$
\frac{\left(d x^{2}+d y^{2}\right)}{y^{2}} .
$$

That is, the hyperbolic cone with cone angle 0 is a hyperbolic cusp. For $\alpha>0$, $N_{\alpha}$ will denote the metric space represented by (2), and $N_{0}$ is that represented by (3).

For $\alpha>0$ one can make the change of variables $\rho=2 \tanh ^{-1}\left(e^{-\alpha y}\right)$ and $\theta=2 \pi x$. One obtains a perhaps more intuitive representation of a hyperbolic cone

$$
d \rho^{2}+\left(\frac{\alpha}{2 \pi}\right)^{2} \sinh ^{2}(\rho) d \theta^{2}
$$

on $\mathbf{R}^{+} \times S^{1}$. The cone point in these "geodesic polar coordinates" is the ideal point $\{0\} \times S^{1}$.

Now one can make precise the notion of a hyperbolic surface with cone points: Let $M$ be a compact surface. Let $g$ be a smooth metric of constant curvature -1 on $M-\left\{c_{i}\right\}_{i=1}^{n}$ where $\left\{c_{i}\right\}_{i=1}^{n}$ is a finite collection of points in $M$. The metric $g$ is a conically singular hyperbolic metric if and only if for each $i=1, \ldots, n$ there exists a neighborhood $U_{i}$ of $c_{i}$ and an isometry $\mu_{i}$ : $U_{i} \rightarrow N_{\alpha_{i}}$ for some $\alpha_{i}$ such that $\mu_{i}\left(c_{i}\right)$ is the cone point. The $c_{i}$ are themselves called cone points with cone angles $\alpha_{i}$, and the $\mu_{i}$ are called (standard) cone charts. Note that all Riemannian complete, finite volume, hyperbolic surfaces are conically singular. In addition, incomplete metrics like the one obtained from the upper half plane quotient of $S L(2, \mathbf{Z})$ fall into this category. The cone points of $\mathbf{H}^{2} / S L(2, \mathbf{Z})$ correspond to the points $\rho, i$, and $\infty$ in the conventional fundamental domain for $S L(2, \mathbf{Z})$.

Note that for a conically singular constant curvature metric $g$ on $M$ the Gauss-Bonet formula takes the form:

$$
\kappa_{g} \cdot \operatorname{Vol}(M, g)+\sum_{i=1}^{n}\left(2 \pi-\alpha_{i}(g)\right)=2 \pi \chi(M)
$$

where $\alpha_{i}(g)$ is the $i^{\text {th }}$ cone angle, $\kappa_{g}$ is the Gaussian curvature, and $\chi(M)$ is the Euler characteristic.

It will be helpful to introduce some notation. Given cone points $\left\{c_{1}, \ldots, c_{n}\right\}$, $\alpha_{i}$ will denote the angle associated to $c_{i} . \vec{\alpha}$ will denote the $n$-tuple $\left(\alpha_{1}, \ldots\right.$, $\left.\alpha_{n}\right)$. To keep track of cusps, a function $V: \mathbf{R}^{n} \rightarrow \operatorname{Power} \operatorname{Set}(\{1, \ldots, n\})$ is 
defined by

$$
V\left(\left(x_{1}, \ldots, x_{n}\right)\right)=\left\{i: x_{i}=0\right\}
$$

Often $V(g)$ will be used as an abbreviation of $V(\vec{\alpha}(g))$.

$\mu$ will denote a standard cone chart into representation (2). Let $C_{a}$ denote $\mu^{-1}\left(S^{1} \times(a, \infty]\right)$ and let $\partial C_{a}$ denote $\mu^{-1}\left(S^{1} \times\{a\}\right) . \partial C_{a}$ will be called a meridian. $\bar{\mu}$ will denote a standard cone chart into representation (4). Let $\bar{C}_{r}$ denote $\bar{\mu}^{-1}\left([0, r) \times S^{1}\right)$ and let $\partial \bar{C}_{r}$ denote $\bar{\mu}^{-1}\left(\{r\} \times S^{1}\right)$. Note that $\partial \bar{C}_{r}$ is a meridian.

Now we make precise the notion of Teichmüller space which shall be used here. Let $M$ be a differentiable surface of genus $g$ and let $\left\{c_{i}\right\}_{i=1}^{n} \subset M$. For $k+l=n$, let $\mathscr{A}_{g, k, l}$ be the set of conically singular metrics $h$ on $\left(M,\left\{c_{i}\right\}_{i=1}^{n}\right)$ with $\vec{\alpha}(h)=\left(\alpha_{1}, \ldots, \alpha_{k}, 0, \ldots, 0\right)$. That is, these metric have at least $l$ cusps. Let the topology of this space be the topology of uniform convergence on compact subsets of $M-\left\{c_{i}\right\}$. Define the interior of $\mathscr{A}_{g, k, l}^{0}$ to be

$$
\mathscr{A}_{g, k, l}^{0}=\left\{h \in \mathscr{A}_{g, k, l}: \alpha_{1}, \ldots, \alpha_{k}>0\right\} .
$$

Let Diff $_{0}$ be the diffeomorphisms $f: M \rightarrow M$ isotopic to the identity which satisfy $f\left(c_{i}\right)=c_{i}$ for each $i=1, \ldots, n$.

Definition 2.1. The Teichmüller space $\mathscr{T}_{g, k, l}$ is defined to be the orbit space $\mathscr{A}_{g, k, l} /$ iff $f_{0}$. Let $\pi: \mathscr{A}_{g, k, l} \rightarrow \mathscr{T}_{g, k, l}$ denote the associated projection. Define the interior of Teichmüller space to be $\pi\left(\mathscr{A}_{g, k, l}^{0}\right)$.

For $h \in \mathscr{A}_{g, k, l}$, let $\mathscr{C}(h)$ denote the metrics in $\mathscr{A}_{g, k, l}$ which are pointwise conformally equivalent to $h$. That is, $h^{\prime} \in \mathscr{C}(h)$ if and only if there exists a function $u: M-\left\{c_{i}\right\}_{i=1}^{n} \rightarrow \mathbf{R}^{+}$such that $h^{\prime}=e^{2 u} h$. In [J] it is shown that each member of $\mathscr{C}(h)$ is determined by its angle $n$-tuple $\vec{\alpha}$.

It is known that a constant curvature metric $g$ induces a compatible complex structure $J(g)$ on $M-\left\{c_{i}\right\}_{i=1}^{n}$. Since $\mu_{i}$ is an isometry, its restriction is holomorphic with respect to this structure. Thus, by adding the charts $\mu_{i}$, one extends $J(g)$ to all of $M$. Further, for each such complex structure there exists a unique complete hyperbolic metric on $M-\left\{c_{i}\right\}_{i=1}^{n}$ such that each $c_{i}$ is a cusp. In particular we obtain a map

$$
p: \mathscr{T}_{g, k, l} \rightarrow \mathscr{T}_{g, 0, k+l}
$$

such that $p^{-1}(\pi(h))=\pi(\mathscr{C}(h))$ for each $h \in \mathscr{A}_{g, k, l}$. From this one finds that $\mathscr{T}_{g, k, l} \hookrightarrow \mathbf{R}^{6 g-6+3 k+2 l}$.

Now consider a path $\gamma:(-\epsilon, \epsilon) \rightarrow \mathscr{A}_{g, k, l}$. For each coordinate neighborhood $U \subset \subset M-\left\{c_{i}\right\}_{i=1}^{n}$ we have

$$
\gamma(t)=\sum_{i, j=1}^{2} \gamma_{i, j}(t) d x_{i} d x_{j} .
$$


The path $\gamma$ is said to be real-analytic iff for each such system of local coordinates we have that each $\gamma_{i, j}:(-\epsilon, \epsilon) \rightarrow C^{2, \alpha}(U)$ is real-analytic. Note that Theorem 3.3 in [J] implies that a path $\gamma:(-\epsilon, \epsilon) \rightarrow \mathscr{C}(h) \subset \mathscr{A}_{g, k, l}$ is real-analytic if and only if $\vec{\alpha}(\gamma)$ is real-analytic. The notion of a real-analytic path naturally descends to $\mathscr{T}_{g, k, l}$ from $\mathscr{A}_{g, k, l}$ via $\pi$.

The following is the key lemma extracted from [J]:

Lemma 2.1 [J]. Let $\gamma:(-\epsilon, \epsilon) \rightarrow \mathscr{C}$ be real-analytic with $\gamma(t)=e^{2 u_{t}} \gamma(0)$. Then

$$
\dot{u}(p)=\sum_{i=1}^{n} \alpha_{i}^{\prime}(0) G_{2}\left(c_{i}, p\right)
$$

where $G_{2}$ is the Green's function associated to $\Delta-2$. If $\alpha_{i}(0)=0$ for each $i$ such that $\alpha_{i}$ is non-constant, then $\dot{u}=0$ and

$$
\ddot{u}=\sum_{i=1}^{n} \frac{\left(\alpha_{i}^{\prime}(0)\right)^{2}}{3} E_{2}^{i}
$$

where $E_{2}^{i}$ is the non-holomorphic Eisenstein series at $s=2$ which is bounded on $C_{b, j}$ for $j \neq i$.

Note that here the Eisenstein series is defined as in [CdV]. In particular, $E_{s}^{i}$ is given by the classical series representation if and only if $(M, g)=\mathbf{H}^{2} / \Gamma$ for some $\Gamma$. If $c_{i}$ corresponds to a cusp at $i \infty$, the classical series representation is

$$
E_{2}^{i}(z)=\sum_{\gamma \in \Gamma / \Gamma_{0}}(\operatorname{Im}(\gamma z))^{2} .
$$

Also in [J] the first variation of the cone charts is computed. Let $\mu_{\gamma(0)}^{-1}$ and $\mu_{\gamma(t)}^{-1}$ be cone charts for $\gamma(0)$ and $\gamma(t)$ respectively. Let $(u, v)$ and $(x, y)$ be the respective coordinates on the corresponding model cones. Then using these coordinates one can write $\mu_{\gamma(t)} \circ \mu_{\gamma(0)}^{-1}=(x(u, v), y(u, v))$. Let $\dot{y}, \dot{x}, \ddot{x}$, and $\ddot{y}$ denote $\frac{d}{d t} x, \frac{d}{d t} y, \frac{d^{2}}{d t^{2}} x$, and $\frac{d^{2}}{d t^{2}} y$ respectively. Let $\dot{\alpha}=\alpha_{i}^{\prime}(0)$. In [J] it is found that if $\alpha_{0} \neq 0$ then

$$
\dot{y}(u, v)=\dot{y}(u, b)+\int_{b}^{v} \dot{h}(u, t) d t
$$

and

$$
\dot{x}(u, v)=\dot{x}(u, b)-\dot{y}_{u}(u, b)-\int_{b}^{v} \int_{b}^{s} \dot{h}(u, t) d t d s
$$

where $\dot{h}=\dot{u}-\dot{\alpha}\left(\frac{1}{\alpha(0)}-\operatorname{coth}\left(\alpha_{i}(t) y\right)\right)$ in $C_{b, i}$. Further, if $\alpha_{i}(0)=0$ for $i$ such that $\alpha_{i}^{\prime}(0) \neq 0$, then one has that

$$
\ddot{y}(u, v)=\ddot{y}(u, b)+\int_{b}^{v} \ddot{h}(u, t) d t
$$


and

$$
\ddot{x}(u, v)=\ddot{x}(u, b)-\ddot{y}_{u}(u, b)-\int_{b}^{v} \int_{b}^{s} \ddot{h}(u, t) d t d s
$$

where $\ddot{h}=\ddot{u}-\frac{(\dot{\alpha})^{2}}{6} y$ in $C_{b, i}$.

\section{Conical PSEUdo-Laplacians}

The balance of this paper is a perturbational study of the Laplace spectrum for conically singular hyperblic metrics with cusps, cone points with zero cone angle. The perturbation of the Laplace spectrum associated to Riemannian complete, hyperbolic metrics is the subject of several recent papers: [CdV], [PS1], [PS2], [Wp2], [Ji], [Pe], and [PS3]. In these studies, the pseudo-Laplacian is the main analytical tool used to deal with the perturbation of embedded eigenvalues. In this section, the pseudo-Laplacian construction is generalized to allow for varying cone angle. The resulting operator is called the conical pseudoLaplacian and is denoted by $\Delta_{S}^{b}$. The basic properties of $\Delta_{S}^{b}$ are discussed, and the spectrum of $\Delta_{S}^{b}$ is shown to be relatively well-behaved under metric perturbation (Corollary 3.1). To link the spectra of the pseudo-Laplacian and the conical pseudo-Laplacian near a discontinuity of $V(\vec{\alpha})$ (i.e. on opening a cusp to a cone) a "coincidence principle" is provided (Lemma 3.1). Finally, all the results on $\Delta_{S}^{b}$ are combined to show that a sequence of cusp forms tends to a cusp form at a discontinuity of $V(\vec{\alpha})$ if the associated eigenvalues limit above $\frac{1}{4}$ (Proposition 3.3).

3.1. Definitions and basic properties. Call a function $b$-cuspidal at the cone point $c$ if and only if for each $a>b$

$$
\int_{\partial C_{a}} f d s=0
$$

where $\partial C_{a}$ is a meridian about $c$ with respect to the cone representation (2). Let $V(\vec{\alpha}(g)) \subset S \subset\{1, \ldots, n\}$. Let $C_{S}^{b}$ denote the smooth compactly supported functions on $M$ which are $b$-cuspidal at $c_{i}$ if $i \in S$. When using (4) these functions will be referred to as $r$-cuspidal functions. These spaces of functions are related by $\tanh \left(\frac{r}{2}\right)=e^{-\alpha b}$ where $\alpha$ is the cone angle.

On $C_{S}^{b}$ the operator $1+\Delta$ is semi-bounded below by 1 , and thus one can apply the method of Friedrichs to obtain a selfadjoint extension of $1+\Delta$ on $\mathscr{L}_{S}^{2, b}$, the closure of $C_{S}^{b}$ with respect to the $L^{2}\left(d V_{g}\right)$ norm [RN], [LP]. This operator will be denoted by $\Delta_{S, g}^{b}$

Because the domain of the pseudo-Laplacian is important to the study of the associated analytic perturbation theory, we sketch here Friedrich's construction: Define the inner product

$$
I_{g}(u, v)=\int_{M}\left(\left\langle\operatorname{grad}_{g}(u), \operatorname{grad}_{g}(v)\right\rangle_{g}+u \cdot v\right) d V_{g}
$$


where

$$
\operatorname{grad}_{g}: C^{1}(M) \rightarrow \Gamma\left(T\left(M-\left\{c_{i}\right\}\right)\right)
$$

is the gradient with respect to the metric tensor $g$. Note that $I_{g}$ is defined on $C_{S}^{b}$. Let $H_{g}$ denote the completion of $C^{\infty}(M)$ with respect to $I_{g}$. The natural identification of $H_{g} \hookrightarrow \mathscr{L}_{S, g}^{2, b}$ is implicit in the following.

The domain of the pseudo-Laplacian is defined to be

$$
D_{S, g}^{a}=\left\{u \in H_{g}: \exists f \in \mathscr{L}_{S, g}^{2, b} \ni I_{g}(u, v)=\int_{M} f \cdot v d V_{g} \forall v \in H_{g}\right\} .
$$

Note that the $f$ in (12) is unique because $v \mapsto \int_{M} f \cdot v d V_{g}$ is a bounded linear functional in the $I_{g}$-norm. We define $\Delta_{S, g}^{b} u$ to be equal to $f-u$. Integration by parts shows that this is consistent with the definition on $C_{S}^{b}$. Equation (11) shows that this operator is selfadjoint.

The positive real-number $b$ will be referred to as the construction parameter. Note that if $S=\left\{i: \alpha_{i}=0\right\}$, then $\Delta_{S}^{b}$ is the usual pseudo-Laplacian $\Delta^{b}$ of [CdV], and if $V=V(\vec{\alpha}(g))=\varnothing$, then $\Delta_{V}^{b}$ is the usual Laplace-Beltrami operator $\Delta_{g}$.

Let

$$
f_{0}^{i}(y)=\int_{\partial C_{y, i}} f d s
$$

denote the zeroeth Fourier coefficient of a continuous function $f: M \rightarrow \mathbf{C}$ about the cone point $c_{i}$. Define the truncation operator $\left(\hat{\bullet}_{S}^{b}\right.$ by

$$
(\hat{f})_{S}^{b}(p)= \begin{cases}f(p), & p \in M-C_{b, i}, \\ f(p)-f_{0}^{i}(y(p)), & p \in C_{b, i} .\end{cases}
$$

To make the exposition relatively self-contained, some important properties of $\Delta_{S}^{b}$ are listed here.

Proposition 3.1. The conical pseudo-Laplacian $\Delta_{S}^{b}$ has the following properties:

(1) $\Delta_{S}^{b}$ is compactly resolved and thus has discrete spectrum.

(2) Functions in the domain of $\Delta_{S}^{b}$ are locally $H^{1}$ except (perhaps) across the meridian $\partial C_{b}$.

(3) Let $T_{f}$ be the distribution associated to $f \in \operatorname{Domain}\left(\Delta^{b}\right)$. Then for each $i \in S$ there exist constants $k_{i}$ such that

$$
\Delta^{b} T_{f}=\Delta T_{f}+\sum_{i \in S} k_{i} Z_{i}^{b}
$$

where $Z_{i}^{b}$ is defined by $Z_{i}^{b}(\phi)=\phi_{0}^{i}(b)$. 
(4) For $f \in C^{2}\left(M-\partial C_{b}\right)$

$$
k_{i}=\lim _{y \rightarrow b^{-}} \frac{\partial}{\partial y} f_{0}^{i}
$$

and $\Delta^{b} T_{f}$ is the distribution given by integrating against $\left.(\Delta f)\right|_{M-\partial C_{b}}$.

(5) The eigenfunctions of $\Delta_{S}^{b}$ are continuous on $M$ and smooth on $M-\partial C_{b}$. Proof. The same as in the case of the usual pseudo-Laplacian. See [LP], [CdV]. Property (4) follows from integration by parts.

A function $f: M \rightarrow \mathbf{C}$ is of moderate growth at the cone point $c$ if and only if there exist constants $B, D$, and $k$ such that for all $b>B$,

$$
|f|_{C^{0}\left(M-C_{b}\right)}<D \cdot b^{k}
$$

where $C_{b}$ denotes a standard neighborhood of $c$. Let $\Delta_{\infty}=-\operatorname{div}(\operatorname{grad})$, the $C^{\infty}$-Laplacian.

Proposition 3.2. A bounded, continuous function $\phi$ is an eigenfunction of $\Delta_{S}^{b}$ if and only if there exists a moderate growth eigenfunction $f$ of $\Delta_{\infty}$ with real eigenvalue such that $\phi=(\hat{f})_{S}^{b}$.

Proof. Proposition 3.1 imples that $(\hat{\phi})_{S}^{b}$ is $C^{\infty}$ on $C^{b^{\prime}}, b^{\prime}<b$. The zeroeth Fourier coefficient of $\phi$ on $\partial C_{a}, b^{\prime}<a<b$, is defined by a second order ODE with real-analytic coefficients. The solution can be extended real-analytically over $C^{b}$ and then added to $\phi$ to result in an eigenfunction $f$ of $\Delta_{\infty}$. That $f$ is of moderate growth is seen from the growth behavior of the zeroeth Fourier coefficient of a $\Delta_{\infty}$ eigenfunction with eigenvalues $s(1-s)$. (See expansions (47) and (49), and estimates (52) and (53).)

3.2. The limiting behavior of eigenfunctions. Next, the limiting behavior of eigenfunctions under metric perturbation is considered. Especially important for applications is the behavior of the spectrum near a discontinuity of $V(\vec{\alpha})$. Recall the space of conically singular metrics $A_{g, k, l}$ defined in Section 2.

Theorem 3.1 (Compactness). Suppose that $\left\{h_{m}\right\}_{m=1}^{\infty}$ converges to $h$ in $\mathscr{A}_{g, k, l}$. For each $m \in \mathbf{Z}$, let $\psi_{m}$ be a moderate growth eigenfunction of $\Delta_{\infty, h_{m}}$ with eigenvalue $\lambda_{m}$. If $\sup \left(\lambda_{m}\right)$ is finite, then there exists a subsequence of $\left\{\psi_{m}\right\}_{m=1}^{\infty}$ which converges smoothly on compact sets to a non-trivial, moderate growth eigenfunction of $\Delta_{\infty, h}$.

Proof. Choose $b_{1}>\sup \left|\lambda_{m}\right|(2 \pi)^{-1}$. We will use the sup norm over $M-C_{b_{1}}$ to normalize the eigenfunctions. In particular, assume that $\left|\psi_{m}\right|_{C^{0}\left(M-C_{b_{1}}\right)}=1$. Thus one has $\left|\psi_{m}\right|_{C^{0}(B)} \leq 1$ where

$$
B=\left\{(x, y): 0 \leq x \leq 1 ; b_{0} \leq y \leq b_{1}\right\} .
$$

This gives a uniform bound on the Euclidean $L^{2}$ norm over $B:\left|\psi_{m}\right|_{L^{2}(B)} \leq 1$. Let $\psi_{m, 0}$ represent the zeroeth Fourier coefficient with respect to polar coordi- 
nates about the cone point. Parseval's formula implies that $\left|\psi_{m}-\psi_{m, 0}\right|_{L^{2}(B)} \leq$ 1. Thus, Lemma A.2 in Appendix A implies that $\psi_{m}-\psi_{m, 0}$ is uniformly bounded. Thus, it will suffice to estimate the growth in the zeroeth Fourier coefficient in each cone point $c_{i}$.

Consider a single cone point $c=c_{i}$. Let $\alpha_{m}$ denote the cone angle of $h_{m}$ at $c . \psi_{m, 0}$ satisfies the differential equation (cf. Appendix)

$$
\frac{\sinh ^{2} \alpha_{m} y}{\alpha_{m}^{2}} \psi_{m, 0}^{\prime \prime}(y)=\lambda_{m} \psi_{m, 0}(y) .
$$

The normalization $\left|\psi_{m}\right|_{C^{0}\left(M-C_{b_{1}}\right)}=1$ implies that $\left|\psi_{m, 0}\right|_{B} \leq 1$. By multiplying both sides of the differential equation by $\psi_{m, 0}^{\prime}$ and integrating over intervals of the form $\left[b_{0}, b\right]$ with $b \leq b_{1}$, one finds a uniform bound on $\left|\psi_{m, 0}^{\prime}\right|_{C^{0}(B)}$. This bound depends on the choices of $b_{0}$ and $b_{1}$, and the hypothesized uniform bound on $\lambda_{m}$.

Thus, equality (13) represents a sequence of ordinary differential equations with bounded coefficients and bounded initial conditions. The elementary theory of such equations gives a subsequence of $\psi_{m, 0}$ which converges uniformly on compact sets. One obtains a uniform bound on $\psi_{m, 0}$ on compact subsets of $C_{b_{0}}-\{$ cone point $\}$. (Note that a subsequence of cone charts $\mu_{m}$ for $h_{m}$ converges to $\mu$ the cone chart for $h$. See [J], Lemma 3.2.) Putting this together with the uniform bound on the other Fourier coefficients, one can construct functions $K(b)$ and $M(b)$ on $\left[b_{0}, \infty\right)$ so that for $m>M(b)$, one has

$$
\left|\psi_{m}\right|_{C^{0}\left(M-C_{b}\right)} \leq K(b) .
$$

A Schauder estimate is used to conclude the proof. The basic estimate is

$$
|\psi|_{C^{2, \beta}\left(M-C_{b^{\prime}}\right)} \leq C_{b^{\prime}, b}\left(\left|\left(\Delta_{\beta}-\lambda\right) \psi\right|_{C^{\beta}\left(M-C_{b}\right)}+|\psi|_{C^{0}\left(M-C_{b}\right)}\right)
$$

for $b^{\prime}<b$ [BJS]. ${ }^{2}$ Here, $C_{b, b^{\prime}}$ does not depend on $\beta$ or $\lambda$ so long as $b$ and $\lambda$ are both bounded. We apply this estimate to the sequence $\psi_{m}$ with $b^{\prime}$, $b>b_{0}$, and obtain

$$
\begin{aligned}
\left|\psi_{m}\right|_{C^{2, \beta}\left(M-C_{b^{\prime}}\right)} & \leq C_{b^{\prime}, b}\left|\psi_{m}\right|_{C^{0}\left(M-C_{b}\right)} \\
& \leq C_{b^{\prime}, b} K(b) .
\end{aligned}
$$

Thus, for a fixed $b$ and $b^{\prime}$ we have a $C^{2, \beta^{\prime}}\left(M-C_{b^{\prime}}\right)$ convergent subsequence where $\beta^{\prime}<\beta$. A diagonalization argument, with for example $b=n$ and $b^{\prime}=n-1$, gives a subsequence which converges $C^{2, \beta^{\prime}}$ on each compact set of $M-\{$ cone point $\} . C^{k}$ estimates for any $k \in \mathbf{Z}^{+}$follow from iteration

\footnotetext{
${ }^{2}$ Note that the ellipticity constant of $\Delta_{\beta}$ tends to zero in the cusp, and therefore $C$ increases as $b$ tends to infinity.
} 
of the Schauder estimate. The limit $\psi_{\infty}$ is non-trivial since by normalization $\left|\psi_{\infty}\right|_{C^{0}\left(M-C_{b_{1}}\right)}=1$.

Remark. The same method of proof applies to the collar pinching case as considered in [Wp2] and [Ji]. From the parallel argument applied to that case, one sees that $\frac{1}{4}$ need not be excluded as a limiting value.

Corollary 3.1. Suppose that $\left\{h_{m}\right\}_{m=1}^{\infty}$ converges to $h$ in $\mathscr{A}_{g, k, l}$. Let $\left(\psi_{m}, \lambda_{m}\right)$ denote an eigenpair of $\Delta_{S, h_{m}}^{b}$. If $\sup \left(\lambda_{m}\right)$ is finite, then a subsequence of $\left\{\psi_{m}\right\}_{m=1}^{\infty}$ converges uniformly on compact subsets of $M-\left\{c_{i}\right\}_{i=1}^{n}$ to an eigenfunction of $\Delta_{S, h}^{b}$.

Proof. Combine Theorem 3.1 and Proposition 3.2.

The following lemma relates the spectra of the conical pseudo-Laplacians and the pseudo-Laplacians near a discontinuity of

$$
V(\vec{\alpha}): \mathscr{A}_{k, l} \rightarrow \text { Power } \operatorname{Set}(\{1, \ldots, n\}) .
$$

In particular, assume that there exists a unique $j$ such that $\alpha_{j}^{\prime}(0) \neq 0$ and $\alpha_{j}(0)=0$. Lemma 3.1 gives the existence of a sequence of angles tending to zero for which the spectrum of the conical pseudo-Laplacian with zeroeth Fourier coefficient truncated in the $j^{\text {th }}$ cone agrees with the spectrum of the conical pseudo-Laplacian whose eigenfunctions are not truncated in the $j^{\text {th }}$ cone. Further, the associated eigenfunctions differ by application of the truncation operator $\left({ }^{*}\right)_{S}^{b}$.

Lemma 3.1 (Coincidence Principle). Let $\gamma:[0,1] \rightarrow \mathscr{A}_{g, k, l}$ be continuous such that $V=V(\vec{\alpha}(\gamma))$ is constant on $(0,1]$ and $\operatorname{Card}(V(\gamma(0)))=C$ ard $(V)+$ 1. Let $(\lambda, \psi):(0,1] \rightarrow \mathbf{R} \times C^{0}(M)$ be an eigenbranch of $\Delta_{\gamma}^{b}$. If there exist $\epsilon, M>0$ such that $\lambda((0,1]) \subset\left[\frac{1}{4}+\epsilon, M\right]$, then there exists a sequence

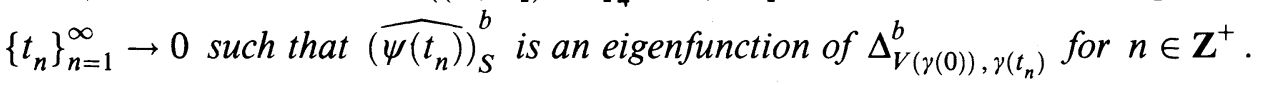
Proof. See Appendix A.3.

Proposition 3.3. Let $\gamma:[0,1] \rightarrow \mathscr{A}_{g, k, l}$ be continuous and as in Lemma 3.1. Suppose further that $(\lambda, \psi):(0,1] \rightarrow\left(\frac{1}{4}, \infty\right) \times C^{\infty}(M)$ is an eigenbranch of $\Delta_{\gamma}$ such that $\lim _{t \rightarrow 0} \lambda(t)=\mu>\frac{1}{4}$. Then there exists a sequence $\left\{s_{n}\right\}_{n=1}^{\infty} \rightarrow 0$ such that $\psi\left(s_{n}\right)$ converges smoothly on compact sets of $M-\left\{c_{i}\right\}_{i=1}^{n}$ to a cusp form of $\Delta_{\gamma(0)}$ with eigenvalue $\mu$.

Proof. $\mu=\frac{1}{4}+r^{2}$ is an eigenvalue of $\Delta^{b}$ associated to a sum of truncated moderate growth eigenfunctions including truncated Eisenstein series if and only if $b^{2 i r}$ is an eigenvalue of the scattering matrix $\Phi\left(\frac{1}{2}+i r\right)$. Choose $b$ so that $b^{2 i r}$ is not an eigenvalue of $\Phi\left(\frac{1}{2}+i r\right)$.

Since $\psi(t)$ is a cusp form, $\psi(t)$ is an eigenfunction of $\Delta^{b}$. Lemma 3.1 gives a sequence $\left\{s_{n}\right\}_{n=1}^{\infty} \rightarrow 0$ such that ${\widehat{\psi\left(t_{n}\right)_{V(\gamma(0))}}}^{b}$ is an eigenfunction of $\Delta_{V(\gamma(0))}^{b}$ 
for $n \in \mathbf{Z}^{+}$. Corollary 3.1 gives a subsequence $\left\{{\widehat{\psi\left(s_{n}\right)}}_{V(\gamma(0))}^{b}\right\}_{n=1}^{\infty}$ converging uniformly on compact sets to an eigenfunction $\psi$ of $\Delta^{b}$. Proposition 3.2 implies that there exists a moderate growth eigenfunction $f$ of $\Delta_{\infty, \gamma(0)}$ with eigenvalue $\mu$ such that $\psi=\widehat{(f)}_{V(\gamma(0))}^{b}$. The choice of $b$ forces $\psi$ to be a cusp form. Smooth convergence follows (for example) from choosing $b$ to be arbitrarily large.

\section{REGULARITY OF EIGENBRANCHES}

Let $\gamma:(-\epsilon, \epsilon) \rightarrow \mathscr{A}_{g, k, l}$ be real-analytic. Are the associated eigenbranches of $\Delta_{S, \gamma}^{b}$ real-analytic? First note that if one assumes that the Phillips-Sarnak conjecture holds true, then this question makes no sense unless one assumes that $V(\vec{\alpha}(\gamma(-\epsilon, \epsilon))) \subset S$. By assuming $V(\vec{\alpha}(\gamma(-\epsilon, \epsilon))) \subset S$, one can show that the eigenbraches are indeed real-analytic. In the present paper we only prove this fact in the case where $\gamma$ is a path in the interior $\mathscr{A}_{g, k, l}^{0}$. In the latter part of this section we consider what happens at a discontinuity of $V(\vec{\alpha}(\gamma))$.

4.1. Analyticity of eigenbranches. The following situation is implicit in the remainder of this section: The path $\gamma:(-\epsilon, \epsilon) \rightarrow \mathscr{A}_{k, l}$ is real-analytic and $V(\vec{\alpha}(\gamma(-\epsilon, \epsilon))) \subset S$. We wish to apply the Kato-Rellich theory of analytic perturbations $[\mathrm{K}]$ to this situation. This theory requires that the family of closed operators be defined on a fixed Hilbert space. It also requires that the domain of each operator does not depend on the perturbation parameter.

For most paths $\gamma:(-\epsilon, \epsilon) \rightarrow \mathscr{A}_{k, l}$, both the Hilbert space $\mathscr{L}_{S, \gamma(t)}^{2, b}$ and the pseudo-Laplacian domain, $D_{S, \gamma(t)}^{b}$, are not constant in $t$. However, one can (non-canonically) construct a family of diffeomorphisms $W_{t}: M \rightarrow M$ such that both $\mathscr{L}_{S, W_{t}^{*}(\gamma(t))}^{2, b}$ and $D_{S, W_{t}^{*}(\gamma(t))}^{b}$ are independent of $t$.

Recall from Section 2 the notation $\mu$ and $\bar{\mu}$ for the respective coordinate charts into the respective cone representations (2) and (4). Let $\hat{M}=M-$ $\left(\cup_{i \notin V(\gamma)} C_{r-1, i}\right) \cup\left(\cup_{i \in V(\gamma)} C_{a-1, i}\right)$. Define the diffeomorphism $W_{t}$ via a partition of unity so that it satisfies the following:

$$
W_{t}(p)= \begin{cases}p, & p \in \hat{M}, \\ \bar{\mu}_{\gamma(t), i} \circ \bar{\mu}_{\gamma(0), i}^{-1}, & p \in C_{r, i}, i \notin V(\gamma(t)), \\ \mu_{\gamma(t), i} \circ \mu_{\gamma(0), i}^{-1}, & p \in C_{a, i}, i \in V(\gamma(t)) .\end{cases}
$$

$W_{t}$ will be dubbed the (meridian) realignment map. In particular $W_{t}$ is constructed to map $\gamma(0)$-meridians to $\gamma(t)$-meridians in a neighborhood of $c_{i}$ for each $i \in S$. Note that since $\mu_{\gamma(t)}$ depends real-analytically on $t, W_{t}$ also depends real-analytically on $t$.

Define a new metric family on $M$ by $\tilde{\gamma}(t)=W_{t}^{*}(\gamma(t))$. Note that $\pi(\tilde{\gamma})=$ $\pi(\gamma)$ where $\pi: \mathscr{A}_{g, k, l} \rightarrow \mathscr{T}_{g, k, l}$ is the projection onto Teichmüller space.

First note that the meridians of the new metric family $\tilde{\gamma}$ do not vary. Hence the space of $r$-cuspidal functions is fixed for this family. Further, from the 
definition of $W_{t}$, one finds that for each $i$ the volume form restricted to $C_{a, i}$ (resp. $C_{r, i}$ ) does not depend on $t$. Thus, since local square-integrability does not depend on the metric one obtains:

Lemma 4.1. If $V(\vec{\alpha}(\gamma))$ is continuous, then the space $\mathscr{L}_{S, \tilde{\gamma}(t)}^{2, a}$ does not depend on $t$.

To be able to apply the Kato-Rellich theory, one must also verify that the domain of $\Delta_{S, \tilde{\gamma}(t)}^{a}$ is independent of $t$. Recall from (12) that the domain of $\Delta_{S, \tilde{\gamma}(t)}^{a}$ is

$D_{S, \tilde{\gamma}(t)}^{a}=\left\{u \in H_{\tilde{\gamma}(t)}: \exists f \in \mathscr{L}_{S, \tilde{\gamma}(t)}^{2, a} \ni I_{\tilde{\gamma}(t)}(u, v)=\int_{M} f \cdot v d V_{\tilde{\gamma}(t)}, \quad \forall v \in H_{\tilde{\gamma}(t)}\right\}$ where $I_{g}$ is the inner product defined by (11).

Lemma 4.2. $D_{\tilde{\gamma}(t)}$ is independent of $t$.

Proof. Suppose $u \in D_{S, \tilde{\gamma}(t)}^{a}$. That is, $u$ represents an $I_{\tilde{\gamma}(t)}$-Cauchy sequence $\left\{u_{k}\right\}_{k=1}^{\infty} \subset C^{\infty}(M)$ such that $f_{n}=\Delta_{\gamma(t), \infty} u_{n}$ converges in $L^{2}$-norm to a function $f$. Integration by parts gives

$$
\left\|g_{n}-g_{m}\right\|_{L^{2}(\tilde{\gamma}(s))}^{2}=I_{\tilde{\gamma}(s)}\left(u_{n}-u_{m}, u_{n}-u_{m}\right) .
$$

Let $\chi_{A}$ denote the characteristic function associated to the set $A \subset M$. The real-analyticity of the metric tensor on compact sets implies that there exists a constant $K$ such that

$$
I_{\hat{\gamma}(s)}\left(\chi_{\hat{M}}\left(u_{n}-u_{m}\right), \chi_{\hat{M}}\left(u_{n}-u_{m}\right)\right) \leq K_{1} \cdot I_{\hat{\gamma}(t)}\left(\chi_{\hat{M}}\left(u_{n}-u_{m}\right), \chi_{\hat{M}}\left(u_{n}-u_{m}\right)\right) \text {. }
$$

By using (4) and (11), one can show that for $\phi$ with support in $C_{r, i}, i \notin V(\gamma)$, we have

$$
\min \left\{\frac{\alpha_{i}(s)}{\alpha_{i}(t)}, \frac{\alpha_{i}(t)}{\alpha_{i}(s)}\right\} I_{\tilde{\gamma}(t)}(\phi, \phi) \leq I_{\tilde{\gamma}(s)}(\phi, \phi) \leq \max \left\{\frac{\alpha_{i}(s)}{\alpha_{i}(t)}, \frac{\alpha_{i}(t)}{\alpha_{i}(s)}\right\} I_{\tilde{\gamma}(t)}(\phi, \phi) .
$$

Note that for $i \in V(\gamma)$ and $\phi$ with compact support in $C_{a, i}$, the quantity $I_{\tilde{\gamma}(s)}(\phi, \phi)$ is constant in $t$. Hence, it follows from (15) and (16) that $\left\{g_{n}\right\}$ is an $L^{2}$-Cauchy sequence. The conclusion follows by noting that $g=\lim _{n \rightarrow \infty} g_{n}$ satisfies

$$
I_{\hat{\gamma}(s)}(u, v)=\int_{M} g \cdot v
$$

for each $v \in H_{\tilde{\gamma}(s)}$.

Remark. Note that the use of geodesic polar coordinates (4) is crucial here. Lemmas 4.1 and 4.2 do not hold if one uses isothermal coordinates like (2). On the other hand, one does not have geodesic polar coordinates if the cone is a cusp, that is, if $\alpha_{i}=0$. In particular, it is impossible to make a satisfactory comparison of the respective norms if $\alpha_{i}(\gamma(s)) \neq \alpha_{i}(\gamma(t))=0$. 
Theorem 4.1. Let $\gamma:(-\epsilon, \epsilon) \rightarrow \mathscr{T}_{g, k, l}^{0}$ be real-analytic with $V(\vec{\alpha}(\gamma(-\epsilon, \epsilon))) \subset$ $S$. Then there exists $\tilde{\gamma}:(-\epsilon, \epsilon) \rightarrow \mathscr{A}_{g, k, l}^{0}$ such that $\pi(\tilde{\gamma})=\gamma$ and the operator family $\Delta_{S, \tilde{\gamma}}^{b}$ is a selfadjoint real-analytic family in the sense of Kato.

Proof. Apply Lemmas 4.1 and 4.2 and note that for each $u \in D_{\tilde{\gamma}(t)}$ and $v \in$ $\mathscr{L}_{S, \gamma}^{2, b}$ the complex-valued function

$$
\int_{M} v \cdot \Delta_{S, \tilde{\gamma}(t)}^{b} u
$$

is real-analytic in $t$.

Using the Kato-Rellich theory ([K], Chapter VII) one obtains as a corollary: Theorem 4.2. Let $\gamma:(-\epsilon, \epsilon) \rightarrow \mathscr{T}_{g, k, l}^{0}$ be real-analytic with $V(\vec{\alpha}(\gamma(-\epsilon, \epsilon))) \subset$ $S$. Then the eigenvalues of the operator family $\Delta_{S, \gamma}^{b}$ can be written as a countable set of real-analytic functions on $(-\epsilon, \epsilon)$. For each such eigenvalue branch, there exist corresponding eigenfunction branches $\psi_{t} \in \mathscr{L}_{S, \gamma(t)}^{2, b}$ such that $W_{t}^{*}\left(\psi_{t}\right)$ is a real-analytic path in $\mathscr{L}_{S, \tilde{\gamma}(0)}^{2, b}$. For a given $t$, the collection of $\psi_{t}$ spans $\mathscr{L}_{S, \gamma(t)}^{2, b}$.

4.2. Estimating the distortion of $W_{t}$. In applications we shall be interested in paths $\gamma:(-\epsilon, \epsilon) \rightarrow \mathscr{C}$ where $\mathscr{C}$ is a (pointwise) conformal equivalence class inside $\mathscr{A}_{g, k, l}$. In this case the associated family of Laplacians is of the form $\Delta_{\gamma(t)}=e^{2 u_{t}} \Delta_{\gamma(0)}$. And thus-at least formally-one can write

$$
\left.\frac{d}{d t}\right|_{t=0} \Delta_{\gamma(t)}=\left.\frac{d}{d t}\right|_{t=0} u_{t} \cdot \Delta_{\gamma(0)} .
$$

This formula simplifies perturbational expressions such as the eigenvalue derivative and the Phillips-Sarnak disappearance condition. Unfortunately, $\Delta_{\gamma(t)}$ is not real-analytic in the sense of Kato in general. Hence, it will be necessary to show that the perturbational expressions derived using $\Delta_{W_{t}^{*}(\gamma(t))}$ are not far off from the ideal expressions obtained from the use of (17).

Let $\gamma:(-\epsilon, \epsilon) \rightarrow \mathscr{C}$ be real-analytic, and let $\Delta_{S, \gamma}^{b}$ denote the associated family of conical pseudo-Laplacians where $V(\gamma(-\epsilon, \epsilon)) \subset S$. Define $\tilde{\gamma}_{t}=$ $W_{t}^{*}\left(\gamma_{t}\right)$ where $W_{t}$ is the realignment map (14). Let $\left(\psi_{t}, \lambda(t)\right)$ be an $\mathscr{L}_{S, \tilde{\gamma}}^{2, b}$ eigenbranch of $\Delta_{S, \tilde{\gamma}_{t}}^{b}$ given by Theorem 4.2. The family $\Delta_{S, \tilde{\gamma}(t)}^{b}$ is real-analytic in the sense of Kato and hence we have an expansion

$$
\Delta_{S, \tilde{\gamma}(t)}^{b}=\Delta_{\gamma(0)}+t A_{b}^{(1)}+t^{2} A_{b}^{(2)}+\cdots
$$

where $A_{b}^{(n)}$ are closed operators on $\mathscr{L}_{S, \gamma(0)}^{2, b}$. Collecting the first order terms of the eigenequation, one has

$$
\left(A_{b}^{(1)}-\dot{\lambda}\right) \psi_{0}+\left(\Delta_{S, \gamma(0)}^{b}-\lambda_{0}\right) \dot{\psi}=0
$$

where $\dot{\lambda}$ and $\dot{\psi}$ are the first order variations in $t$ of $\lambda_{t}$ and $\psi_{t}$ respectively. The perturbational quantities which are of interest in the present article are 
obtained by integrating both sides of (19) against a specific moderate growth eigenfunction $\phi$. By integrating the resulting expression by parts one obtains

$$
\int_{M}\left(A_{b}^{(1)} \psi_{0}\right) \phi d V=\dot{\lambda} \int_{M} \psi \cdot \phi d V+\sum_{i \in S}(\phi)_{0, i}(b) \partial_{y}(\dot{\psi})_{0, i}(b) .
$$

Note that in deriving (20) one uses Lemma A.6 to show that the boundary terms vanish at the cone point.

The following lemma shows that the perturbational effect of the realignment map $W_{t}$ is negligible. Let $\dot{W}$ denote the $t$-derivative of the maps $W_{t}$ at $t=0$.

Lemma 4.3. Let $W_{t}$ be the meridian realignment map defined by (14). Let $S_{k}=\{(x, y): k<y<k+2 ; 0 \leq x \leq 1\}$. Then for each $i \in\{1, \ldots, n\}$

$$
\sup _{k>b}\left|\dot{W} \circ \mu_{i}^{-1}\right|_{C^{2, \alpha}\left(S_{k}\right)}
$$

is finite.

Proof. It suffices to consider $\dot{W}$ (resp. $\ddot{W}$ ) on the cone neighborhoods $C_{r, i}$ for which

$$
W_{t}(p)=\bar{\mu}_{\gamma(t), i}^{-1} \circ \bar{\mu}_{\gamma(0), i} .
$$

From equations (7) and (8) one sees that it is enough to have $C^{3, \alpha}$ estimates on $\dot{h}$. But $\dot{h}$ is an $L^{2}$ eigenfunction with trivial Fourier coefficient in $C_{b}$ for some $b$. Hence, the desired estimates follow from Lemma A.4 in Appendix A.

The following is the key lemma which shows that the difference between the ideal quantities and those derived from the technically necessary realignment are as close as one likes for large $b$.

Lemma 4.4. Suppose $\gamma:(-\epsilon, \epsilon) \rightarrow \mathscr{C}$ is real-analytic with $V(\gamma(t))$ continuous and $\gamma(t)=e^{2 u_{t}} \gamma(0)$. Let $\psi_{t}$ be an eigenbranch of $\Delta_{\tilde{\gamma}(t)}$ and let $\phi$ be a moderate growth eigenfunction of $\Delta_{\infty, \gamma(0)}$. Then

$$
\lim _{b \rightarrow \infty} \int\left(A_{b}^{(1)} \psi\right) \phi=-2 \lambda_{0} \int \dot{u} \phi \psi_{0} .
$$

Proof. The usual Laplacian and the pseudo-Laplacian agree below $b$. In particular, for functions $f$ with support in $M-C_{b}$, one has $\Delta_{S, \tilde{\gamma}(t)}^{b} f=\Delta_{\gamma(t)} f$. Hence for such functions

$$
\begin{aligned}
A_{b}^{(1)} f & =\frac{d}{d t} e^{-2 u_{t}} \Delta_{\gamma_{0}} f \\
& =-2 \dot{u} \Delta_{\gamma_{0}} f
\end{aligned}
$$

where $\dot{u}=\frac{d}{d t} u$. Thus it suffices to show that the integral $\int_{C_{b}}\left(A_{b}^{(1)} \psi\right) \phi$ tends to zero as $b$ tends to infinity. Since $\psi$ is a cusp form, $\Delta_{S, \tilde{\gamma}(t)}^{b} \psi=\Delta_{\tilde{\gamma}(t)} \psi$. But 
$\Delta_{\tilde{\gamma}(t)} \psi=\left(W_{t}^{*}\right)^{-1} \circ \Delta_{\gamma(t)} W_{t}^{*}$. It follows that

$$
A^{(1)} \psi=\left(\dot{W}_{t}^{*}\right)^{-1} \circ \Delta_{\gamma(0)} \psi-2 \dot{u} \Delta_{\gamma(0)} \psi+\Delta_{\gamma(0)}\left(\psi \circ \dot{W}_{t}\right) .
$$

By Lemma 4.3, $\dot{W}_{t}$ and its derivatives up to the second order are bounded. Since the volume form decays exponentially in $y, \int_{C_{b}}\left(A_{b}^{(1)} \psi\right) \phi$ tends to zero as desired.

4.3. Discontinuities of $V(\alpha)$ : Asymptotic perturbation theory. Now we turn to the case where $\gamma:(-\epsilon, \epsilon) \rightarrow \mathscr{A}_{g, k, l}$ is real-analytic and $V(\vec{\alpha}(\gamma))$ has a discontinuity at 0 . One can show that if $V(\gamma(-\epsilon, \epsilon)) \subset S$, then the eigenvalues $\Delta_{S, \gamma}^{a}$ can be written as a countable union of real-analytic functions. Further, one can show that the corresponding eigenfunctions vary real-analytically in $C^{0}(K)$ for $K \subset \subset M-\left\{c_{i}\right\}_{i=1}^{n}$. Unfortunately, the proof of this fact is rather laborious and will not be given here.

However, in applications the real-analyticity of eigenvalues and eigenfunctions is not needed. We only need to obtain the analogue of Lemma 4.4, that is Lemma 4.9. To do this it will suffice to use asymptotic perturbation ([K], Chapter 8). This method is as laborious as showing real-analyticity, but the method has the added bonus of showing that the second derivative of an eigenfunction branch belongs to $L^{2}$. From this latter fact, one can obtain the bounds which are crucial in the proof of Lemma 4.9.

Let $\gamma:(-\epsilon, \epsilon) \rightarrow \mathscr{C}$ be real-analytic with $\alpha_{j}(\gamma(t))$ identically zero for some $j$. In addition to the assumption $S \supset V(\vec{\alpha}(\gamma(t)))$ for all $t$, assume that for all $i$ for which $\alpha_{i}^{\prime}(t) \neq 0$, one has $i \in S$ and $\alpha_{i}(0)=0$. In [J] it is shown that in this case the formal Taylor expansion of the conformal factor in $\alpha$ about $\alpha=0$ has only even terms. Hence the additional assumption implies that the formal power series of $\gamma(t)$ about $t=0$ has only even terms.

Asymptotic perturbation theory requires a fixed Hilbert space, with an operator family of the form $T_{t}=T+t \cdot T^{(1)}$. In [Wp2], S. Wolpert has slightly generalized the theory to a family of the form $T_{t}=T+t \cdot T^{(1)}+B_{t}$ on a fixed Hilbert space with appropriate conditions on the size of $B_{t}$. This generalization is what shall be used here.

Just as before, the operators $\Delta_{S, \gamma(t)}^{b}$ do not act on a fixed Hilbert space and thus modifications are required. Note however that the realignment map $W_{t}$ defined in (14) is not well defined at a discontinuity of $V(\gamma)$. But the following analogous family is well defined at a discontinuity. Let $J_{t}: M \rightarrow M$ be defined via a partition of unity to satisfy the following:

$$
W_{t}(p)= \begin{cases}p, & p \in \hat{M}, \\ \mu_{\gamma(t), i} \circ \mu_{\gamma(0), i}^{-1}, & p \in C_{a, i}, \quad i \in\{1, \ldots, n\} .\end{cases}
$$

Just as with $W_{t}$, the family $J_{t}$ maps meridians to meridians. Thus, the metric family $\tilde{\gamma}=J_{t}^{*}(\gamma)$ has a fixed family of $b$-cuspidal functions. 
Recall that by assumption $\gamma(t)$ is an even function of $t$. Hence $J_{t}$ is an even function of $t$, and thus $\tilde{\gamma}(t)$ is also even in $t$.

Unfortunately, the volume forms $d V_{\tilde{\gamma}(s)}$ and $d V_{\tilde{\gamma}(t)}$ are inequivalent unless $\vec{\alpha}(\gamma(s))=\vec{\alpha}(\gamma(t))$. Hence the associated spaces $\mathscr{L}_{S, \gamma(t)}^{2, b}$ in general depend on $t$. To resolve this, one conjugates the family $\Delta_{S, \tilde{\gamma}}^{b}$ with a family of bijective multiplication operators $\Phi_{t}: \mathscr{L}_{S, \gamma(t)}^{2, b} \rightarrow \mathscr{L}_{S, \gamma(0)}^{2, b}$. The $\Phi_{t}$ are defined via a fixed partition of unity to satisfy

$$
\left(\Phi_{t} f\right)(p)= \begin{cases}f(p), & p \in M-\cup_{i=1}^{n} C_{a, i}, \\ \frac{\alpha_{i}(t) y(p)}{\sinh \left(\alpha_{i}(t) y(p)\right)} f(p), & p \in C_{a-1, i}, \quad i \in\{1, \ldots, n\} .\end{cases}
$$

Asymptotic perturbation theory will be applied to the operator family $T_{s}$ : $\mathscr{L}_{S, \tilde{\gamma}(0)}^{2, b} \rightarrow \mathscr{L}_{S, \tilde{\gamma}(0)}^{2, b}$ defined by

$$
T_{s} f=\Phi_{\sqrt{s}} \Delta_{S, \tilde{\gamma}(\sqrt{s})}^{b} \Phi_{\sqrt{s}}^{-1} .
$$

Note that since $\tilde{\gamma}(t)$ and $\Phi_{t}$ are even functions of $t, T_{s}$ is well defined.

Since $W_{t}, \gamma(t)$, and $\Phi_{t}$ vary real-analytically in $C^{\infty^{s}}(K)$ for compact sets $K \in M-\left\{c_{i}\right\}_{i=1}^{n}$, and the pseudo-Laplacian only involves finitely many derivatives, one has for each $f \in C^{\infty}(K)$ the $C^{\infty}(K)$-convergent expansion

$$
T_{s} f=T^{(0)} f+s T^{(1)} f+s^{2} T^{(2)} f+s^{3} T^{(3)} f+\cdots .
$$

Define

$$
B_{s}=T_{s}-T^{(0)}-s T^{(1)} .
$$

These operators are extended to $C^{\infty}\left(M-\left\{c_{i}\right\}_{i=1}^{n}\right)$ by choosing a compact exhaustion. Then for $f \in C^{\infty}\left(M-\left\{c_{i}\right\}_{i=1}^{n}\right)$ we have $T_{s} f=T^{(0)} f+s T^{(1)} f+B_{s} f$. In the application of the theory it will be useful to restrict $T^{(1)}$ and $B_{s}$ to the subspace

$$
\begin{array}{r}
\mathscr{E}=\left\{f \in \mathscr{L}_{S}^{2, b} \cap C^{\infty}\left(M-\left\{c_{i}\right\}_{i=1}^{n}\right):\left|D^{\beta}(f)\right|_{C^{0}\left(C_{a, i}\right)} \leq C e^{-2 \pi a},\right. \\
|\beta| \leq 2, i \in\{1, \ldots, n\}\} .
\end{array}
$$

In particular define the domains of $B_{s}$ and $T^{(1)}$, denoted $D\left(B_{s}\right)$ and $D\left(T^{(1)}\right)$ respectively, to be $\mathscr{E}$.

An application of asymptotic perturbation theory requires the verification of several hypotheses. We refer the reader to Chapter VIII of [K], Sections 1 and 2, and [Wp2], Section 3.

(1) $T_{s}$ converges strongly in the generalized sense to $T^{(0)}$.

(2) The region of boundedness non-trivially intersects the resolvent set $P\left(T^{(0)}\right)$.

(3) $D\left(B_{s}\right)$ is $t$-independent.

(4) Both $T^{(0)}$ and $T_{s}$ are closed operators on $X$.

(5) $D\left(T^{(0)}\right) \cap D\left(T^{(1)}\right) \cap D\left(B_{s}\right)$ is a core for $T^{(0)}$. 
(6) For each $u \in D\left(B_{s}\right)$ there exists a constant $C$ such that $\left\|B_{s}(u)\right\| \leq C s^{2}$.

Lemma 4.5. Conditions (1) through (6) are satisfied by the operator family defined by equation (25).

Proof. Conditions (3) and (4) are clearly satisfied. It is claimed that $\mathscr{E}$ is a core for $T^{(0)}$. To see this, note that $\mathscr{E}$ contains finite sums of eigenfunctions of $T_{0}$. [K], Problem III.5.19, implies that $\mathscr{E}$ is a core for $T_{0}=T^{(0)}$. (Note that $\mathscr{E}$ is a core for $T_{0}$ if and only if $\mathscr{E}$ is a core for $T_{0}-\lambda, \lambda \in P\left(T^{(0)}\right)$.) Thus, condition (5) is satisfied.

It is easy to verify that for each $u \in \mathscr{E}, T_{s} u$ tends to $T_{0} u$ in $\mathscr{L}_{S, \gamma(0)}^{2, b}$-norm as $s$ tends to zero. Thus, condition (1) follows from [K], Corollary VIII.1.6. Condition (2) also follows from [K], Corollary VIII.1.6.

Condition (6) remains to be checked. Suppose $\alpha_{i}(t)$ is nonconstant. Then by assumption $\alpha_{i}(0)=0$ and $i \in S$. Let

$$
Q_{i, s}(y)=\frac{\sinh \alpha_{i}(\sqrt{s}) y}{\alpha_{i}(\sqrt{s}) y} .
$$

Let $\Delta_{E u c}$ denote the Laplacian for the standard flat metric on the cylinder $C_{b, i}$. For functions $f$ whose support is contained in $C_{b, i}$ one finds

$$
T_{s} f=Q_{i, s}(y) \Delta_{E u c}\left(Q_{i, s}(y) f\right)
$$

$$
=\left(Q_{i, s}(y)\right)^{2} \Delta_{E u c} f+Q_{i, s}(y) \frac{d}{d y}\left(Q_{i, s}(y)\right) \partial_{y} f+Q_{i, s}(y) \frac{d^{2}}{d y^{2}}\left(Q_{i, s}(y)\right) f .
$$

If $f$ also belongs to $\mathscr{E}$, then it follows that the series expansion (26) converges in $\mathscr{L}_{S, \tilde{\gamma}(0)}^{2, b}$-norm. Condition (6) follows.

Let $\Lambda_{\tilde{\gamma}(t)}$ denote the distinct eigenvalues of $\Delta_{S, \tilde{\gamma}(t)}^{b}$. Let $V_{\lambda}$ denote the eigenspace associated to $\lambda$.

Lemma 4.6. Let $\lambda$ be an eigenvalue of the operator $\Delta_{S, \tilde{\gamma}(0)}^{b}$. Given $0<\eta<$ $\operatorname{dist}\left(\lambda, \Lambda_{\tilde{\gamma}(0)}-\{\eta\}\right)$ there exists $\delta>0$ such that if $t \in(-\delta, \delta)$, then

$$
\sum_{\mu \in(\lambda-\eta, \lambda+\eta) \cap \Lambda_{\bar{\gamma}(t)}} \operatorname{dim}\left(V_{\mu}\right)=\operatorname{dim}\left(V_{\lambda}\right) .
$$

Proof. The proof is an application of the minimax principle and Lemma 3.1. We need to normalize the domain of the Dirichlet functional

$$
D_{t}(f)=\frac{\left\langle\Delta_{S, \gamma(t)}^{b} f, f\right\rangle_{\gamma(t)}}{\langle f, f\rangle_{\gamma(t)}} .
$$


To this end, let $\chi_{i} \in C^{\infty}(M)$ such that $\chi_{i}\left(M-C_{a, i}\right)=\{0\}$ and $\chi\left(C_{a, i}\right)=\{1\}$. Define the bijection $A_{t}: \mathscr{L}_{S, \tilde{\gamma}(0)}^{2, b} \rightarrow \mathscr{L}_{S, \tilde{\gamma}(t)}^{2, b}$ by

$$
A_{t}(f)=f \cdot \sum_{i=1}^{n} \chi_{i} \cdot \mu_{i}^{*}\left(\exp \left(y\left(\alpha_{i}(\tilde{\gamma}(t))-\alpha_{i}(\tilde{\gamma}(0))\right)\right)\right)
$$

Then the Dirichlet functional can be reinterpreted as $D_{t}^{*}(f)=D_{t}\left(A_{t}(f)\right)$. Note that $D_{t}^{*}$ is well defined on the space $\mathscr{W} \subset \cap_{t} \mathscr{L}_{S, \tilde{\gamma}(t)}^{2, b}$ of functions $f$ for which

(1) $f$ is $C^{2}$ on the complement of $\left(\cup_{i \in S} \partial C_{b, i}\right) \cup\left(\left\{c_{i}\right\}_{i=1}^{n}\right)$.

(2) $f$ is continuous on $M-\left\{c_{i}\right\}_{i=1}^{n}$.

(3) $f \circ \mu_{i}$ is of rapid decay for each $i \in S$.

Note that if $\phi$ is an eigenfunction of $\Delta_{S, \tilde{\gamma}}^{b}$ then $A_{t}(\phi) \in \mathscr{W}$ and all minima of $D_{t}^{*}$ are of this form.

Let $k_{\text {min }}\left(k_{\text {max }}\right)$ be the limit inferior (superior) of

$$
N(t)=\sum_{\mu \in[0, \lambda+\eta) \cap \Lambda_{\tilde{\gamma}(t)}} \operatorname{dim}\left(V_{\mu}\right)
$$

as $t$ tends to zero. Let $\left\{t_{k}\right\}$ be a sequence tending to zero such that

$$
\lim _{k \rightarrow \infty} N\left(t_{k}\right)=k_{\text {max }}
$$

Using Lemma 3.1 one can construct a subsequence $t_{k_{j}}$ and families of orthonormal eigenfunctions $\left\{\psi_{i}\left(t_{k_{j}}\right)\right\}_{i=1}^{N\left(t_{k_{j}}\right)}$ with eigenvalues in $[0, \lambda+\eta)$ such that $\lim _{j \rightarrow \infty} \psi_{i}\left(t_{k_{j}}\right)$ is an eigenfunction $\psi_{i}(0)$ of $\Delta_{S, \tilde{\gamma}(0)}^{b}$. Because the volume forms $d V_{\gamma(t)}$ are $t$-continuous, the $\psi_{i}(0)$ are all orthogonal. Further, the continuity in $t$ of $D_{t}^{*}$ implies that for each $i=1, \ldots, k_{\text {max }}$, the eigenvalue of $\psi_{i}(0)$ lies in $[0, \lambda+\eta)$. It follows that $k_{\max } \leq N(0)$.

Let $\left\{s_{k}\right\}$ be a sequence tending to zero for which $N\left(s_{k}\right)=k_{\text {min }}$. Choose $k_{\text {min }}$ orthonormal subsequences of eigenfunctions $\left\{\psi_{i}\left(s_{k_{j}}\right)\right\}_{i=1}^{k_{\min }}$ such that $\lim _{j \rightarrow \infty} \psi_{i}\left(s_{k_{j}}\right)$ is an eigenfunction $\psi_{i}(0)$ of $\Delta_{S, \tilde{\gamma}}^{b}$. Minimize the functional $D_{s_{k_{j}}}^{*}$ on the subspace $U_{j}=\mathscr{W} \ominus\left(\bigoplus_{i=1}^{k_{\min }} \mathbf{C} \cdot \psi_{i}(0)\right)$. The continuity of the functional $D^{*}$ and the convergence of the spaces $\mathscr{U}_{j}$ imply that the minimum value converges to the minimum value of $D_{0}^{*}$ on $\mathscr{W} \ominus\left(\bigoplus_{i=1}^{k_{\min }} \mathbf{C} \cdot \psi_{i}(0)\right)$. Thus the minimum value is larger than $\lambda+\eta$. Hence $k_{\text {min }} \geq N(0)$.

The conclusion of the lemma follows from induction with respect to $\Lambda_{\gamma(0)}$.

Proposition 4.1. Let $\gamma:(-\epsilon, \epsilon) \rightarrow \mathscr{C}$ be real-analytic. Let $\tilde{\gamma}(t)=W_{t}^{*}(\gamma(t))$. Suppose that $S \supset V(\gamma(t))$ for all $t$. Also suppose that $\alpha_{i}(\gamma(t))$ is non-constant if 
and only if $\alpha_{i}(0)=0$. Let $\left(\lambda_{t}, \psi_{t}\right)$ be an eigenbranch of $\Delta_{S, \tilde{\gamma}(t)}^{b}$. Then $\left.\frac{d^{2}}{d t^{2}}\right|_{t=0} \psi_{t}$ belongs to $\mathscr{L}_{S, \gamma(0)}^{2, b}$.

Proof. The theorem will follow from [K], Theorem VIII.2.6 (more precisely, its generalization in Section 3, [Wp2]) applied to the family $T_{s}$. In particular, note that there is a one-to-one correspondence between eigenbranches $\phi_{s}$ of $T_{s}$ and the eigenbranches $\psi_{t}$ of $\Delta_{S, \tilde{\gamma}(t)}^{b}$ by $\psi_{\sqrt{s}}=\phi_{s}$. In particular we have:

$$
\left.\frac{d^{2}}{d t^{2}}\right|_{t=0} \psi_{t}=\left.\frac{d}{d s}\right|_{s=0} \phi_{s} .
$$

The hypotheses of [K], Theorem VIII.2.6, have already been verified in Lemmas 4.5 and 4.6. In particular, the stability of the eigenvalues follows from Lemma 4.6.

Let $\ddot{\psi}$ denote the left-hand side of $(30)$.

Lemma 4.7. The function $\ddot{\psi}$ and its derivatives up to second order are $O\left(e^{-y}\right)$ at each cone point.

Proof. Consider the eigenequation restricted to a cone $c_{i}$ for which the function $\alpha_{i}(\gamma(t))$ is non-trivial:

$$
\frac{\sinh \alpha_{i}(t) y}{\alpha_{i}(t) y} \Delta_{\alpha_{i}(0)} \psi_{t}=\lambda_{t} \psi_{t} .
$$

Differentiating twice at $t=0$ one obtains

$$
\left(\Delta_{0}-\lambda_{0}\right) \ddot{\psi}=\left(y^{2}-\ddot{\lambda}\right) \psi_{0} .
$$

Since $\psi_{0}$ has trivial zeroeth Fourier coefficient, the zeroeth Fourier coefficient of $\dot{\psi}$ is of the form $A y^{s}+B y^{1-s}$ where $s=\frac{1}{2}+i r$. But Proposition 4.1 implies that $\ddot{\psi}$ belongs to $L^{2}\left(y^{-2} d x d y\right)$. Hence, $\ddot{\psi}$ has trivial zeroeth Fourier coefficient in this cone. Lemma A.7 applies to give a $C^{0} O\left(e^{-y}\right)$ estimate on $\ddot{\psi}$. To obtain a $C^{2}$ estimate, a Schauder estimate is applied. In particular let $S_{m}=\{(x, y): 0 \leq x \leq 1 ; m \leq y \leq m+1\}$. We have

$$
\left(\Delta_{E u c}-\lambda y^{-2}\right) \ddot{\psi}=\left(1-\ddot{\lambda} y^{-2}\right) \psi_{0} .
$$

Applying a Schauder estimate with respect to the elliptic operator $\left(\Delta_{E u c}-\lambda y^{-2}\right)$ one obtains

$$
|\psi|_{C^{2, \alpha}\left(S_{m}\right)} \leq C\left(\left|\left(1-\ddot{\lambda} y^{-2}\right) \psi_{0}\right|_{C^{\alpha}\left(S_{m}\right)}+|\ddot{\psi}|_{C^{0}\left(S_{m}\right)}\right) .
$$

Since the constant $C$ depends only on the ellipticity constant, the shape of the domain, and the bounds on the coefficients, $C$ can be chosen to be independent of $m$. The proof is complete.

Now we are ready to apply the above analysis to derive the analogue of Lemma 4.4. Note that the expansion $(18)$ is not valid in the $\mathscr{L}_{S, \tilde{\gamma}}^{2, b}$ sense 
because the operator family in this case is not real-analytic in the sense of Kato. But $\Delta_{\tilde{\gamma}(t)}$ is real-analytic on $C^{0}(K)$ for compact $K \subset M-\left\{c_{i}\right\}_{i=1}^{n}$. Note also that by assumption the family $\tilde{\gamma}(t)$ is even in $t$ and hence we have an expansion

$$
\Delta_{\tilde{\gamma}(t)}=\Delta_{\gamma(0)}+t^{2} A_{b}^{(2)}+\cdots
$$

where the operators $A_{b}^{(2 n)}$ act on $C^{\infty}$ functions on $M-\left\{c_{i}\right\}_{i=1}^{n}$. By adding the distribution $\sum_{i \in S} k_{i} Z_{i}^{b}$, one obtains

$$
\Delta_{S, \tilde{\gamma}(t)}^{b}=\Delta_{S, \gamma(0)}^{b}+t^{2} A_{b}^{(2)}+\cdots .
$$

Collecting the second order terms of the eigenequation, one has

$$
\left(A_{b}^{(2)}-\ddot{\lambda}\right) \psi_{0}+\left(\Delta_{S, \gamma(0)}^{b}-\lambda_{0}\right) \ddot{\psi}=0 .
$$

Next, integrate both sides of (32) against a moderate growth eigenfunction $\phi$. By integrating the resulting expression by parts one obtains

$$
\int_{M}\left(A_{b}^{(2)} \psi_{0}\right) \phi d V=\ddot{\lambda} \int_{M} \psi_{0} \cdot \phi d V+\sum_{i \in S}(\phi)_{0, i}(b) \partial_{y}(\ddot{\psi})_{0, i}(b) .
$$

Note that in deriving (33) one uses Lemma 4.7 to show that the boundary terms vanish at the cone point.

In order to use (33) we need the analogue of Lemma 4.4. First we estimate the effect of the diffeomorphism $J_{t}$.

Lemma 4.8. Let $J_{t}$ be the map defined by (24). Let $S_{k}=\{(x, y): k<y<$ $k+2 ; 0 \leq x \leq 1\}$. Then

$$
\sup _{k>b}\left|\ddot{J} \circ \mu_{i}^{-1}\right|_{C^{2, \alpha}\left(S_{k}\right)}
$$

is finite.

Proof. The proof is the same as for Lemma 4.3 except that one uses estimates (9) and (10).

Lemma 4.9. Suppose $\gamma:(-\epsilon, \epsilon) \rightarrow \mathscr{C}$ is real-analytic with $V(\gamma(t))$ continuous except at $t=0$ and $\gamma(t)=e^{2 u_{t}} \gamma(0)$. Let $\psi_{t}$ be an eigenbranch of $\Delta_{\tilde{\gamma}(t)}$ and let $\phi$ be a moderate growth eigenfunction of $\Delta_{\infty, \gamma(0)}$. Then

$$
\lim _{b \rightarrow \infty} \int\left(A_{b}^{(2)} \psi\right) \phi=-2 \lambda_{0} \int \ddot{u} \phi \psi_{0} .
$$

Proof. The proof differs from the proof of Lemma 4.4 only in that the volume form does not decay rapidly in $y$ and that $W_{t}$ is replaced by $J_{t}$. However, the cusp form $\psi$ and its derivatives do decay rapidly in $y$. Thus, Lemma 4.8 provides the conclusion. 


\section{MoNOTONICITY}

In this section we consider the behavior of the eigenvalue branches whose existence is guaranteed by Theorem 4.2. We find that under certain circumstances the eigenvalues vary directly with the size of the cone angle. In particular, any eigenvalue branch of the usual Laplacian is an increasing function of the cone angle (Theorem 5.1). As a corollary one finds that the eigenvalues associated to the Neumann and Dirichlet problems on geodesic triangles in the hyperbolic plane vary monotonically with respect to the measure of a vertex angle (Theorem 5.1). The basic idea behind the proof is the use of the first variational term of the metric given by Lemma 2.1 .

Recall that $V(\alpha(\gamma))$ is the set of cusps, i.e. cones with cone angle zero.

Theorem 5.1. Suppose $\gamma:(-\epsilon, \epsilon) \rightarrow \mathscr{C}$ is a non-trivial real-analytic metric deformation such that $V(\alpha(\gamma))$ is continuous on $(-\epsilon, \epsilon)$ and such that for each $i=1, \ldots, n$ the function $\left.\alpha_{i}(\gamma(t))\right)$ is non-decreasing. Let $\lambda:(-\epsilon, \epsilon) \rightarrow \mathbf{R}$ denote an $L^{2}$ eigenbranch associated to $\Delta_{\gamma}$. Then $\lambda$ is a strictly increasing function.

Proof. Without loss of generality, all derivatives are evaluated at $t=0$; otherwise reparametrize. Let $\psi_{t}$ denote an $L^{2}$ eigenfunction branch associated to $\lambda(t)$. Note that $\psi_{t}$ is an eigenbranch of $\Delta_{V(\gamma), \gamma}$ for all $b$. By choosing $\left\|\psi_{0}\right\|=1$, equation (20) becomes

$$
\dot{\lambda}=\int\left(A_{b}^{(1)} \psi_{0}\right) \psi_{0} .
$$

Since $V(\alpha(\gamma(t)))$ is continuous, and thus constant, $\lambda$ is independent of $b$. Hence, Lemma 4.4 implies that

$$
\dot{\lambda}=-2 \lambda_{0} \int \dot{u}\left(\psi_{0}\right)^{2}
$$

Since $V(\alpha(\gamma))$ is assumed to have no discontinuities, Lemma 2.1 implies that $\dot{u}=\sum_{i=1}^{n} k_{i} \dot{\alpha}_{i} G_{2}\left(c_{i}, \cdot\right)$ where $G_{2}$ is the Green's function associated to $-\Delta-2$. A maximum principle argument shows that $G_{2}\left(c_{i}, \cdot\right) \leq 0$. And since $\psi_{0}^{2}, G_{2}$, and the deformation are nontrivial, it follows that $\dot{\lambda}>0$.

Remark. Using an argument similar to that found in Theorem 5.1, one can show that there exists $b^{*}$ such that for all $b>b^{*}$ the eigenvalues of the conical pseudo-Laplacians, $\Delta_{S, \gamma}^{b}$, have monotone eigenbranches. Note however that $b^{*}$ might depend on $t$.

Corollary 5.1. Let $T$ and $T^{\prime}$ be geodesic triangles in $\mathbf{H}^{2}$ with cone angle triples $\left(\alpha_{1}, \alpha_{2}, \alpha_{3}\right)$ and $\left(\beta_{1}, \beta_{2}, \beta_{3}\right)$ such that $\alpha_{i}>\beta_{i}>0$ for $i=1,2$, and 3 . Arrange the respective eigenvalues of the Dirichlet and Neumann problems in increasing order. Then a Dirichlet eigenvalue of $T$ is greater than the corresponding Dirichlet eigenvalue of $T^{\prime}$, and a Neumann eigenvalue of $T$ is greater than the corresponding Neumann eigenvalue of $T^{\prime}$. 
Proof. By gluing two copies of $T$ together and two copies of $T^{\prime}$ together, one obtains two conically singular metrics $\left(S^{2}, h\right)$ and $\left(S^{2}, h^{\prime}\right)$, with cone angle $n$ tuples $\left(2 \alpha_{1}, 2 \alpha_{2}, 2 \alpha_{3}\right)>\left(2 \beta_{1}, 2 \beta_{2}, 2 \beta_{3}\right)>\overrightarrow{0}$ respectively. Since there is only one conformal structure on $S^{2}, h$ and $h^{\prime}$ belong to the same conformal class $\mathscr{C}=\mathscr{C}\left(S^{2}, J,\left\{c_{i}\right\}_{i=1}^{3}\right)$. From the uniformization theorem, Theorem 2.2 in [J], one finds that the set of cone angle $n$-tuples which represents $\mathscr{C}$ is convex in $\mathbf{R}^{3}$. Thus one can choose a linear path $\gamma:(0,1) \rightarrow \mathscr{C}$ joining $\vec{\alpha}(h)$ to $\vec{\alpha}\left(h^{\prime}\right)$.

Note that since all cone angles are positive, the $L^{2}$ spectrum is discrete and hence varies analytically (Theorem 4.2). Theorem 5.1 applies to show that the $k^{\text {th }}$ eigenvalue of $\Delta_{h}$ is greater than the $k^{\text {th }}$ eigenvalue of $\Delta_{h^{\prime}}$. Each $g \in \mathscr{P}$ has an involutive isometry $l_{g} \in \operatorname{Diffeo}\left(S^{2}\right)$ which interchanges the isometric triangles in the triangle decomposition This isometry divides the spectrum into $l$-even and $l$-odd eigenfunctions, or equivalently Neumann and Dirichlet eigenfunctions on the triangles. Since $l_{\gamma}:[0,1] \rightarrow \operatorname{Diffeo}\left(S^{2}\right)$ is continuous, the odd/even labeling is constant on each eigenbranch. It follows that the respective Neumann eigenvalues and the respective Dirichlet eigenvalues of $T$ and $T^{\prime}$ compare as desired.

Remarks. (1) The monotonicity of Dirichlet eigenvalues can be seen from domain monotonicity. But the monotonicity of Neumann eigenvalues is more subtle. For example, there are Sturm-Liouville operators on the real line for which domain monotonicity does not hold for Neumann eigenvalues [GB].

(2) The corollary can be generalized to any pair of conformally equivalent geodesic polygons in $\mathbf{H}^{2}$ with uniformly comparable vertex angles.

(3) The monotonicity had been foreshadowed by the numerical evidence in Tables 1 through 4 in [H2].

In [Sa1], P. Sarnak showed that the eigenvalues associated to the Hecke triangle groups are greater than $\frac{1}{4}$. Here Theorem 5.1 is used to improve this estimate for the odd eigenvalues.

Corollary 5.2. The odd-eigenvalues associated to the Hecke triangle groups are uniformly bounded from below by $\frac{1}{2} \pi^{2}$.

Proof. The hyperbolic surfaces associated to these groups are members of $\gamma$ : $[0,1] \rightarrow \mathscr{C}$ where $\vec{\alpha}(\gamma(t))=(0, \pi, t)$. In particular, $\left(S^{2}, \gamma\left(\frac{2 \pi}{k}\right)\right)=\mathbf{H}^{2} / G_{k}$ where $G_{k}$ is a Hecke triangle group.

As in the proof of Corollary 5.1, for each $t$ there is an involutive isometry $l_{\gamma(t)}: S^{2} \rightarrow S^{2}$ which induces a decomposition into $l$-even and $l$-odd eigenfunctions. The Eisenstein series are $l$-even. Thus, the $l$-odd eigenfunctions of $\Delta_{\{1\}, \gamma(t)}^{b}$ are precisely the $l$-odd eigenfunctions of $\Delta_{\gamma(t)}$. In particular, the $l$-odd eigenfunctions of $\Delta_{\gamma(t)}$ persist though the deformation and form real-analytic branches. Lemma 6.8 and Proposition 3.3 combine to imply that each $l$-odd eigenfunction branch converges to a cusp form. The classical lower bound on $H / \Gamma_{0}(2)$ implies that the limiting value of the eigenvalue is at least $\frac{1}{2} \pi^{2}[\mathrm{H} 1]$. The proposition now follows from Theorem 5.1. 
Remark. In fact, using Theorem 5.1 one finds that any triangle with angle triple $\left(\theta_{1}, \theta_{2}, \theta_{3}\right)>\left(0, \frac{\pi}{2}, 0\right)$ has Dirichlet eigenvalues bounded below by $\frac{1}{2} \pi^{2}$.

\section{THE INSTABILITY OF CUSP FORMS}

In this section the perturbation results developed in the previous sections are used to give evidence which lends weight to the view that the discrete spectrum lying above $\frac{1}{4}$ is unstable under metric perturbation. The evidence consists of Theorems 6.1 and 6.2.

Definition 6.1. Given a metric deformation $\gamma:(\epsilon, \epsilon) \rightarrow \mathscr{A}_{g, k, l}$, define a stable eigenbranch to be an eigenbranch of $\Delta_{\gamma}$ which is well defined and real-analytic for all $t$ (including $t=0$ ). A stable eigenfunction of $\Delta_{\gamma(t)}$ is the evaluation of some stable eigenbranch at $t$.

The following lemma is standard in the perturbation theory of the Laplace spectrum of finite volume hyperbolic surfaces [PS1], [PS3].

Lemma 6.1. Let $\gamma:(\epsilon, \epsilon) \rightarrow \mathscr{A}_{g, k, l}$ be real-analytic. For all but countably many $t$, the set of eigenfunctions of $\Delta_{\gamma(t)}$ with eigenvalues greater than $\frac{1}{4}$ is the union of the stable eigenfunctions and the finite set of eigenbranches whose eigenvalues limit to $\frac{1}{4}$ as $t$ tends to a singularity of $V(\gamma(t))$.

Proof. The eigenfunctions of $\Delta_{\gamma(t)}$ are eigenfunctions of the pseudo-Laplacian if and only if the zeroeth Fourier coefficient vanishes. The zeroeth Fourier coefficient is a real-analytic function and hence vanishes for all $t$ or for only countably many $t$. In the former case, we have a stable eigenvalue provided that the eigenvalue does not limit to $\frac{1}{4}$ as one nears a singularity of $V(\vec{\alpha}(t))$ (Proposition 3.3). General arguments show that only finitely many eigenvalues limit to $\frac{1}{4}$ (see [Wp1]). The lemma follows from these considerations.

Hence, the strategy is to demonstate the nonexistence of stable eigenbranches for some real-analytic deformation.

6.1. Nonsingular deformations. Consider a real-analytic deformation $\gamma:(-\epsilon, \epsilon)$ $\rightarrow \mathscr{C}$ such that $V(\vec{\alpha}(\gamma))$ is continuous. This deformation is nonsingular in the sense that the multiplicity of the continuous spectrum remains constant.

First we deduce the analogue of the disappearance condition found in [PS1], [Wp2], [PS3]. Let $(\psi(t), \lambda(t))$ be an eigenbranch of a family of conical pseudoLaplacians $\Delta_{S, \gamma}^{b}$. Let $\mathbf{E}_{s}$ denote a linear combination of Eisenstein series with $s(1-s)=\lambda(0) . \mathbf{E}_{s}$ is a moderate growth eigenfunction and since $\int \mathbf{E}_{s} \psi(0)=0$ we have from (20)

$$
\int_{M}\left(A_{b}^{(1)} \psi(0)\right) \mathbf{E}_{s} d V=\sum_{i \in S}(\mathbf{E})_{0, i}(b) \partial_{y}(\dot{\psi}(0))_{0, i}(b)
$$

where $(\mathbf{E})_{0, i}(b)$ and $(\dot{\psi})_{0, i}(b)$ denote the respective zeroeth Fourier coefficients associated to the meridian $\partial C_{i, a}$ in the $i^{t h}$ cone. Using (36) and Lemma 4.4 one obtains: 
Lemma 6.2 (Phillips-Sarnak Disappearance Condition). Let $\gamma:(-\epsilon, \epsilon) \rightarrow \mathscr{C}$ such that $V(\gamma)$ is continuous. Let $\mathbf{E}_{s}$ be a linear combination of Eisenstein series and let $\psi(t)$ be an eigenbranch of $\Delta_{V(\gamma), \gamma}^{b}$. If

$$
\int_{M} \dot{u} \psi \mathbf{E}_{s} d V \neq 0
$$

then $\psi$ is not a stable eigenbranch.

Let $\mathscr{T}_{g, n, m}$ be the Teichmüller space of hyperbolic metrics on a genus $g$ surface with $n$ conical singularities and $m$ cusps. A generic point is a point belonging to a residual set in $\mathscr{T}_{g, n, m}$.

Theorem 6.1. If there exists a metric in $\mathscr{T}_{g, n-1, m}$ whose Laplacian has only simple embedded eigenvalues, then the Laplacian of the generic point in $\mathscr{T}_{g, n, m}$ has no square-integrable eigenfunctions with eigenvalue greater than $\frac{1}{4}$. In particular, the Laplacian of the generic point has only finitely many $L^{2}$ eigenfunctions. Proof. Choose a conically singular hyperbolic metric $h$ on $M$ belonging to $\mathscr{T}_{g, n-1, m}$ which has only simple eigenvalues. Let $\left\{c_{i}\right\}_{i=1}^{n-1}$ denote the cone points of $h$. Note that by puncturing $M$ at some point $c$ one has that $h$ also belongs to $\mathscr{T}_{g, n, m}$ with cone angle $2 \pi$ at $c$. In particular, $h=\gamma_{c}(0)$ where $\gamma_{c}:(-\epsilon, \epsilon) \rightarrow \mathscr{C}(h)$ is defined by $\vec{\alpha}(\gamma(t))=\left(t, \alpha_{1}(h), \ldots, \alpha_{n-1}(h)\right)$. We claim that for $c$ in a residual set, $\Delta_{\gamma_{c}}$ has no stable eigenbranches with eigenvalue greater than $\frac{1}{4}$. Note that $V\left(\gamma_{c}\right)$ is continuous and hence eigenfunctions which limit to $\frac{1}{4}$ are stable. Therefore the theorem will follow from the claim and Lemma 6.1.

To prove the claim, let $\psi$ be a cusp form associated to $h$ with simple eigenvalue $\lambda$. Let $\mathbf{E}_{s}$ be a linear combination of Eisenstein series whose zeroeth Fourier coefficient is null at $b$ except in a single cusp $c_{l}$. Both $\psi$ and $\mathbf{E}_{s}$ are real-analytic by elliptic regularity, and hence so is $(-\Delta+2)^{-1}\left(\psi \mathbf{E}_{s}\right)$. Thus the set $W_{\psi}=\left\{w \in M:(-\Delta+2)^{-1}\left(\psi \mathbf{E}_{s}\right)(w) \neq 0\right\}$ is open and dense. Let $W$ be the intersection $\cap W_{\psi_{j}}$ over a (countable) basis of eigenfunctions $\left\{\psi_{j}\right\}$ for the discrete spectrum of $\Delta_{h}$. Note that $W$ is residual and does not depend on $c$.

Let $\psi_{c}(t)$ be a stable eigenbranch of $\Delta_{\gamma_{c}}$. Because $\lambda$ is simple, $\psi=\psi_{c}(0)$ is independent of $c$ (up to a scalar).

Write $\gamma(t)=e^{2 u_{c}(t)} h$. According to Lemma 2.1, $\dot{u}_{c}$ is the Green's function associated to $-\Delta+2$. Hence,

$$
\int_{M} \dot{u}_{c} \psi \mathbf{E}_{s} d V=(-\Delta+2)^{-1}\left(\psi \mathbf{E}_{s}\right)(c)
$$

For $c \in W,(37)$ is nonzero. Thus, Lemma 6.2 implies that $\psi(0)$ is not stable and the claim is proven.

Remarks. (1) Note that many hyperbolic surfaces have eigenvalues less than $\frac{1}{4}$; these do not disappear after perturbation. 
(2) If $m>0$, then $\mathscr{T}_{g, n-1, m}$ is empty unless either $g>1$ or $m+n>$ 3. In particular, the only non-trivial Teichmüller spaces of conically singular hyperbolic metrics not covered by Theorem 6.1 are $\mathscr{T}_{0,1,2}, \mathscr{T}_{0,2,1}$, and those without cusps. ${ }^{3}$ Note that $\mathscr{T}_{0,2,1}$ is covered by Corollary 6.1 .

(3) The reason for the assumption of simplicity: Although an eigenspace for $\Delta_{\gamma_{c}(0)}$ does not depend on $c$, it might be the case that 'the' Kato basis for $\Delta_{\gamma_{c}}^{b}$ has non-trivial dependence on $c$. However, if $\left\{\psi_{j}(0)\right\}_{j=1}^{m}$ is a Kato basis for an eigenspace $V$ of $\Delta_{\gamma(0)}$, then the 'linear form' on the left-hand side of (37) cannot vanish for all $\psi_{i}$ and all $c$. In particular, the subspace of stable eigenfunctions is a proper subspace of $V$ for some $c$. (See the proof of Lemma 6.6.)

6.2. Singular perturbations: The Hecke triangle deformation. If $V(\vec{\alpha}(\gamma(t)))$ has a discontinuity at 0 and no other cone angles vary, then $\dot{u}, \dot{\lambda}$, and $\dot{\psi}$ are all trivial. In this case (36) yields no information. However, from (33) one has

$$
\int_{M}\left(A_{b}^{(2)} \psi\right) \mathbf{E}_{s} d V=\sum_{i \in S}(\mathbf{E})_{0, i}(b) \partial_{y}(\ddot{\psi})_{0, i}(b) .
$$

The following lemma is analogous to Lemma 6.2.

Lemma 6.3. Suppose $\gamma:(-\epsilon, \epsilon) \rightarrow \mathscr{C}$ is real-analytic, and $V(\gamma(t))$ is continuous except at $t=0$, with $\{n\}=V(\gamma(0))-V\left(\gamma\left(\frac{\epsilon}{2}\right)\right)$. Let $\phi$ be a stable eigenfunction of $\Delta_{\gamma(0)}$. Let $\mathbf{E}_{s}$ be a linear combination of Eisenstein series with $s(1-s)=\lambda(0)$. If

$$
\int_{M} \ddot{u} \phi \mathbf{E}_{s} d V \neq 0
$$

then $\left(\mathbf{E}_{s}\right)_{0, n}(b)$, the zeroeth Fourier coefficient in the $n^{\text {th }}$ cone, does not vanish for any $b$.

Proof. Let $\phi(t)$ be the eigenbranch of $\Delta_{\gamma}$ with $\phi(0)=\phi$. Lemma 6.4 implies that there exists an eigenbranch $\psi(t)$ of the operator family $\Delta_{V(\gamma(0)), \gamma}^{b}$ such that $\psi(0)=\phi$. Note that for each $i \in V\left(\gamma\left(\frac{\epsilon}{2}\right)\right), \partial_{y}(\dot{\phi})_{0, i} \equiv 0$. Thus, the Coincidence Principle, Lemma 3.1, implies that $\partial_{y}(\dot{\psi})_{0, i} \equiv 0$ for each such $i$. Hence equation (38) reduces to

$$
\int_{M}\left(A_{b}^{(2)} \phi\right) \mathbf{E}_{s} d V=(\mathbf{E})_{0, n}(b) \partial_{y}(\ddot{\psi})_{0, n}(b) .
$$

Lemma 4.9 together with the hypothesis implies that for $b$ large enough, the left-hand side of $(40)$ is non-zero. But note that if $\left(E_{s}\right)_{0, n}(b)=C \cdot b^{s}+D \cdot b^{1-s}$ vanishes for some $b$, then it vanishes on a set accumulating at $+\infty$.

\footnotetext{
${ }^{3}$ In the latter case the spectrum of the Laplacian is always discrete and hence is of no interest with regard to the Roelcke-Selberg Conjecture.
} 
Recall that a Kato basis for an eigenspace is an orthonormal basis $\left\{\psi_{j}(0)\right\}_{i=1}^{m}$ where each $\psi_{j}(t)$ is continuous as a function of $t$ and where $\psi_{j}(t)$ is an eigenfunction of $\Delta_{\gamma(t)}$ for each $t$.

Lemma 6.4. Suppose $\gamma:(-\epsilon, \epsilon) \rightarrow \mathscr{C}$ is real-analytic with $V(\gamma(t))$ continuous except at $t=0$. Further suppose that $\left.V(\gamma(0))-V\left(\gamma\left(\frac{\epsilon}{2}\right)\right)\right)$ is a singleton. Then any stable eigenfunction of $\Delta_{\gamma}(0)$ is a member of a Kato basis with respect to the operator family $\Delta_{V(\gamma(0)), \gamma}^{b}$.

Proof. Apply the Coincidence Principle, Lemma 3.1.

Now we consider the stability of eigenfunctions associated to a specific family of metrics on the thrice punctured sphere. The Hecke triangle deformation $H:[0, \pi) \rightarrow \mathscr{C}\left(S^{2}, J,\left\{c_{1}, c_{2}, c_{3}\right\}\right)$ is defined so that $H(t)$ has cone angles $(0, \pi, t)$. (Recall that the conformal structure $J$ on $S^{2}$ is unique.) The name Hecke triangle deformation is motivated by the fact that $\mathbf{H}^{2} / G_{k}=\left(S^{2}, H\left(\frac{\pi}{k}\right)\right)$ where $G_{k}$ is a Hecke triangle group [H1]. Note that $\left(S^{2}, H(0)\right)=\mathbf{H}^{2} / \Gamma_{0}(2)=$ $\mathbf{H}^{2} / \Theta$ where $\Gamma_{0}(2)$ is a congruence subgroup of level 2 and $\Theta$ is the Theta group [H1], [T].

The groups $G_{k}, k=3,4,6$, and $\infty$, are arithmetic and hence are expected to have discrete spectrum accounting for Weyl's Law. However, for $k>6, G_{k}$ is not arithmetic, and hence the conjecture of Sarnak [Sa2] predicts that $G_{k}$ has no associated discrete spectrum. Here, an attempt is made to support this conjecture by applying the perturbational strategy to $H(t)$ at $t=0$.

Note that $V(\vec{\alpha}(H(t)))$ has a discontinuity at $t=0$. In particular, Lemma 2.1 gives that $\ddot{u}$ is the Eisenstein series $E_{2}$ associated to the cusp $c_{1}$ on $\left(S^{2}, H(0)\right)=\mathbf{H}^{2} / \Gamma_{0}(2)$. By 'unfolding' the left-hand side of (38) one obtains

$$
\int_{M} \ddot{u} \phi \mathbf{E}_{s} d V=\frac{45 r}{2 \pi^{5} \sinh \pi r} \operatorname{Re}\left(L_{\phi * \mathbf{E}_{s}}(2)\right)
$$

where $L_{\psi_{0} * \mathbf{E}_{s}}(2)$ is the Rankin-Selberg convolution product at $s=2$ of $\psi_{0}$ and $\mathbf{E}_{s}$ having eigenvalue $\lambda=\frac{1}{4}+r^{2}$. See [Wp2], Lemma 2.4.

Recall the Hecke theory concerning Maass cusp forms and Eisenstein series associated to a congruence subgroup of $S L(2, \mathrm{Z})$ ([G], [T]). Let $f \mid A_{2}$ denote $f: \mathbf{H}^{2} \rightarrow \mathbf{C}$ composed with the Mobius transformation $z \mapsto 2 \cdot z$ acting on $\mathbf{H}^{2}$. In this section, functions on $\mathbf{H}^{2} / \Gamma_{0}(2)$ are not distinguished from their liftings to $\mathbf{H}^{2}$. Let $E_{s}$ be the Eisenstein series associated to $S L(2, \mathbf{Z})$. Recall that $E_{s}$ and $E_{s} \mid A_{2}$ span the vector space of Eisenstein series associated to $\Gamma_{0}(2)$. Let $E_{s}^{1}$ denote $E_{s}$ and let $E_{s}^{2}$ denote $E_{s} \mid A_{2}$.

Recall that for each Hecke eigenform $f$ associated to $S L(2, \mathbf{Z})$ there are two linearly independent Hecke eigenforms on $\Gamma_{0}(2): f$ and $f \mid A_{2}$. These are the so-called oldforms. The other Hecke eigenforms on $\Gamma_{0}(2)$ are called newforms. Recall that the Hecke eigenforms provide a basis for the space of Maass cusp forms on congruence subgroups. 
Recall from Section 5 the reflection $\tau$ which induces a division of the spectrum of $\Delta_{H(t)}$ into even and odd eigenfunctions. Given an eigenspace $V$ of $\Delta_{H(0)}$, let $V^{\text {even }}$ denote the subspace of even eigenfunctions. Recall that the Hecke eigenforms are also divided into those which are 'even' or 'odd' with respect to $\tau$. Let $\left\{f_{i}\right\}_{i=1}^{m}$ denote the Hecke basis for $V^{\text {even }}$. Define the $2 \times m$ matrix:

$$
B_{b}=\left(\int_{M}\left(A_{b}^{(2)} f_{i}\right) E^{j}\right)_{i j}
$$

Lemma 6.5 (Wolpert [Wp2]). $B_{\infty}=\lim _{b \rightarrow \infty} B_{b}$ exists and each entry of $B_{\infty}$ is non-zero.

Proof. See [Wp2]. One uses Lemma 4.9 together with the arithmetic properties of Hecke eigenforms. In particular, $L_{f * E}$ can be written as an Euler product since the Fourier coefficients of $f$ and $E$ are multiplicative. $s=2$ belongs to the region of convergence and hence the Euler product expansion is valid there. One checks that the factors are non-zero.

Unfortunately, the Hecke basis for an eigenspace $V$ is not necessarily a Kato basis, the basis on which the disappearance condition must be evaluated. Let $\left\{\psi_{j}^{b}\right\}_{j=1}^{m}$ denote the Kato basis for $V^{e v e n}$ associated to the operator family $\Delta_{S, H}^{b}$. Note that the Kato basis depends on $b$ whereas the Hecke basis does not.

Lemma 6.6. Let $V$ be an eigenspace of $\Delta_{H(0)}$ containing even eigenfunctions. Then at least one (even) eigenfunction in $V$ is not stable.

Proof. Suppose to the contrary that the subspace of even eigenfunctions is spanned by stable eigenfunctions. Then the stable eigenfunctions form a Kato basis which does not depend on $b$. Hence the matrix $U^{b}$ which changes the Hecke basis to the Kato basis has an invertible limit $U_{\infty}=\lim _{b \rightarrow \infty} U_{b}$. Lemma 6.5 gives that both column vectors, $\vec{v}_{1}$ and $\vec{v}_{2}$, of $B_{\infty}$ are nonzero. Hence $U_{\infty} \vec{v}_{1}$ is nontrivial. Thus, for some $i, \int_{M} \ddot{u} \psi_{i} E_{s}^{1} \neq 0$. But $\left(E_{s}^{1}\right)_{0,3}=$ $(2 b)^{s}+\phi(s)(2 b)^{1-s}$ does vanish since $|\phi(s)|=1$ and $\operatorname{Re}(s)=\frac{1}{2}$. Lemma 6.3 provides a contradiction.

At present Lemma 6.6 is the most one can say about the instability of eigenvalues for hyperbolic surfaces without making assumptions about multiplicities. The following hypothesis, although believed to be valid, is not as yet known to be true. (Compare with [Wp2] and [PS3].)

Hypothesis 6.1. The eigenvalues associated to $S L(2, \mathbf{Z})$ and the newform eigenvalues associated to $\Gamma_{0}(2)$ are simple. Further, these sets of eigenvalues are disjoint.

Assuming Hypothesis 6.1 one has from Lemma 6.6 that the even newforms are not the limits of eigenbranches of $\Delta_{H}$. The hypothesis implies that oldform 
eigenspaces are at most 2-dimensional. Let $U_{b}$ be the change of basis matrix between the Hecke basis $\left\{f_{1}, f_{2}\right\}$ and the Kato basis $\left\{\psi_{1}^{b}, \psi_{2}^{b}\right\}$.

Lemma 6.7 (Wolpert [Wp2]). Assume Hypothesis 6.1. Let $V$ be an oldform eigenspace of $\mathbf{H}^{2} / \Gamma_{0}(2)$. Then $\operatorname{Det}\left(B_{\infty}\right) \neq 0$ and $U_{\infty}=\lim _{b \rightarrow \infty} U_{b}$ exists.

Proof. The proof rests on calculations in [Wp2]. There are two basic facts concerning normalized Hecke eigenforms and Eisenstein series which are used. First, $L_{f \mid A_{2} * g}=k L_{f * g}$ where $k$ is an explicit constant depending only on the $2^{\text {nd }}$ Fourier coefficients of $f$ and $g$. Second, one has the standard estimate $\left|c_{2}\right| \leq 2^{\frac{1}{2}}+2^{\frac{-1}{2}}$ where $c_{2}$ is the $2^{\text {nd }}$ Fourier coefficient for a normalized Hecke eigenform. The determinant of $B_{\infty}$ is explicitly calculated in [Wp2].

The change of basis matrix $U_{b}$ is indirectly studied by considering the linear transformation $P_{V} \circ A_{b}^{(2)}: V \rightarrow V$ where $P_{V}$ is the $L^{2}$ projection onto $V$. Note that if the eigenvalues of $P_{V} \circ A_{b}^{(2)}$ are distinct, then $U_{b}$ is also the change of basis matrix for $P_{V} \circ A_{b}^{(2)}$ between the Kato basis and Hecke basis. Lemma 4.9 implies that the matrix representation of $P_{V} \circ A_{b}^{(2)}$ with respect to the Hecke basis $\left\{f, f \mid A_{2}\right\}$ converges to a matrix $D$. Using the facts noted at the beginning of the paragraph one shows that eigenvalues of $D$ are distinct.

Lemma 6.8. Eigenbranches of $\Delta_{H}$ do not limit to $\frac{1}{4}$.

Proof. Suppose not. Then Theorem 3.1 would imply that a subsequence of the associated cusp forms converged to a moderate growth eigenfunction on $H(0)$ with eigenvalue $\frac{1}{4}$. The number $\frac{1}{4}$ is not an eigenvalue of a cusp form on $H(0)=\Gamma_{0}(2)$ [H1]. The scattering matrix $\Phi(s)$ for $\Gamma_{0}(2)$ at $s=\frac{1}{2}$ is equal to $-I d$. Thus, the moderate growth eigenfunctions associated to $\frac{1}{2}$ are $D_{1}=\left.\frac{d}{d s}\right|_{s=\frac{1}{2}}\left(E_{1}\right)(s)$ and $D_{3}=\left.\frac{d}{d s}\right|_{s=\frac{1}{2}}\left(E_{3}\right)(s)$. The matrix of zeroeth Fourier coefficients of these eigenfunctions is

$$
\begin{aligned}
& \left(\begin{array}{cc}
\left(D_{1}\right)_{0,1}(b) & \left(D_{1}\right)_{0,2}(b) \\
\left.\left(D_{3}\right)_{0,1}(b)\right) & \left(D_{3}\right)_{0,2}(b)
\end{array}\right) \\
& \quad=\left(\begin{array}{cc}
\Phi_{11}^{\prime}\left(\frac{1}{2}\right) y^{\frac{1}{2}}+2 \log (y) y^{\frac{1}{2}} & \Phi_{12}^{\prime}\left(\frac{1}{2}\right) y^{\frac{1}{2}} \\
\Phi_{21}^{\prime}\left(\frac{1}{2}\right) y^{\frac{1}{2}} & \Phi_{22}^{\prime}\left(\frac{1}{2}\right) y^{\frac{1}{2}}+2 \log (y) y^{\frac{1}{2}}
\end{array}\right) .
\end{aligned}
$$

On page 571 in $[\mathrm{H} 1], \Phi$ is given explicitly. A calculation shows that the $s$ derivative of each entry of $\Phi(s)$ is nonzero at $s=\frac{1}{2}$. It follows that the zeroeth Fourier coefficients are non-trivial and respectively linearly independent. But, the limiting zeroeth Fourier coefficient must be identically zero in the cusp at $c_{1}$, and hence identically zero in the cusp at $c_{2}$. Contradiction.

Theorem 6.2. Assuming Hypothesis 6.1, at most a countable number of metrics in the Hecke triangle deformation $H(t)$ have even cusp forms associated to them.

Proof. Lemma 6.1 together with Lemma 6.8 implies that it is enough to show that there are no stable eigenbranches. Lemma 6.4 implies that each stable 
eigenfunction is a member of a Kato basis. Lemma 6.7 implies that each row vector of $U_{\infty} B_{\infty}$ has a nonzero entry. That is, for each member of a Kato basis $\psi_{i}$, there is a $j$ such that $\int_{M} \ddot{u} \psi_{i} E^{j} d V \neq 0$. By inspecting the explicit form of $\left(E^{j}\right)_{0,3}$, one finds that it has zeroes. Hence Lemma 6.3 implies that none of the $\psi_{i}$ are stable.

Corollary 6.1. Assuming Hypothesis 6.1, the generic thrice punctured sphere having a conically singular hyperbolic metric with exactly one null cone angle has no associated even eigenfunctions.

Proof. The eigenfunction branches of $\Delta_{\{1\}}^{b}$ vary real-analytically in $\mathscr{T}_{g, k, l}^{0}$ by Theorem 4.2. Thus, the zeroeth Fourier coefficients vary real-analytically in $\mathscr{T}_{g, k, l}^{0}$. The corollary follows from Theorem 6.2.

Recalling the bijection between hyperbolic triangles and thrice punctured spheres one immediately has the following:

Corollary 6.2. Assuming Hypothesis 6.1, the generic hyperbolic triangle with exactly one null angle has no associated Neumann eigenvalues.

One can weaken Hypothesis 6.1 so that one obtains a conclusion which contradicts the assertion that every surface is essentially cuspidal. Recall that a surface is essentially cuspidal if and only if its cusp form eigenvalues account for the full Weyl-Selberg asymptotics [PS3]. We provide a statement of this below in the form of Theorem 6.3.

Let $\Lambda_{g}$ denote the distinct eigenvalues of the $\Delta_{g}$ and

$$
\Lambda_{g}(R)=\Lambda_{g} \cap[0, R) .
$$

$V_{\lambda}$ will denote the eigenspace associated to $\lambda$. Define

$$
\Lambda_{g}^{\leq m}=\left\{\lambda \in \Lambda_{g}: \operatorname{dim}\left(V_{\lambda}\right) \leq m\right\} .
$$

Recall that a surface is essentially cuspidal if and only if its cusp form eigenvalues account for the full Weyl-Selberg asymptotics [PS3].

Theorem 6.3. Let $g$ be the metric for $\mathbf{H}^{2} / \Gamma_{0}(2)$ where $\Gamma_{0}(2)$ is the level 2 congruence subgroup. If

$$
\liminf _{R \rightarrow \infty} \frac{1}{R} \sum_{\Lambda_{\bar{g}}^{\leq m}(R)} \operatorname{dim}\left(V_{\lambda}\right)>0,
$$

then there exists $t_{0}>0$ such that for only a countable set of $t \in\left(0, t_{0}\right)$ is $H(t)$ essentially cuspidal.

Proof. The proof is similar to that of the main result in [PS3]. In particular, one uses Lemma 6.6 and the monotonicity of the eigenvalues (Theorem 5.1).

Remarks. (1) All but finitely many of $\mathbf{H}^{2} / G_{k}$ belong to the set $\{H(t): 0<t<$ $\left.t_{0}\right\}$. 
(2) The lack of a uniform upper bound on the logarithmic derivative of the eigenvalue branches precludes a global statement.

\section{APPENDIX A. FOURIER EXPANSION IN A CONE AND ASYMPTOTICS}

A.1. The differential equation and special functions. Let $L_{\alpha}$ denote the $C^{\infty}$ Laplacian associated to the model hyperbolic cone (3). Then

$$
L_{\alpha}=\frac{\sinh ^{2}(\alpha y)}{\alpha^{2}}\left(\frac{\partial^{2}}{\partial x^{2}}+\frac{\partial^{2}}{\partial y^{2}}\right) .
$$

In this appendix we will consider the Fourier coefficients of the eigenfunctions of $L_{\alpha}$. The eigenfunction equation in cylindrical coordinates is

$$
\frac{\sinh ^{2}(\alpha y)}{\alpha^{2}}\left(\partial_{x}^{2}+\partial_{y}^{2}\right) \psi(x, y)=\lambda \psi(x, y) \text {. }
$$

Separation of variables $\psi(x, y)=w(x) v(y)$ yields

$$
\frac{-w_{x x}}{w}=k=\frac{v_{y y}}{v}-\frac{\lambda \alpha^{2}}{\sinh ^{2} \alpha y}
$$

where $k$ is a constant. Since solutions of the left-hand side of equation (44) must have period 1 , they are spanned by $e^{2 \pi i n x}$ with $k=4 \pi^{2} n^{2}$. Thus, the function $v$ must satisfy

$$
\alpha^{-2}(\sinh (\alpha y))^{2}\left(\partial_{y}^{2} v+4 \pi^{2}|n|^{2} v\right)=s(1-s) v
$$

for some $n \in \mathbf{Z}$ and where $\lambda=s(1-s)$.

If $\alpha=0$, (45) reduces to

$$
y^{2}\left(\partial_{y}^{2} v+4 \pi^{2}|n|^{2} v\right)=s(1-s) v
$$

If $n=0$, a pair of linearly independent solutions to (46) is given by $y^{s}$ and $y^{1-s}$ if $s \neq \frac{1}{2}$, and by $y^{\frac{1}{2}}$ and $y^{\frac{1}{2}} \log (y)$ if $s=\frac{1}{2}$. For $n \neq 0$ a pair of independent solutions is given by $\mathbf{K}_{s}^{n}(y)=y^{\frac{1}{2}} K_{s-\frac{1}{2}}(2 \pi|n| y)$ and $\mathbf{I}_{s}^{n}(y)=y^{\frac{1}{2}} I_{s-\frac{1}{2}}(2 \pi|n| y)$ where $I$ and $K$ are the Bessel functions of imaginary argument [Le]. Thus, the expansion of an eigenfunction of $L_{0}\left(s \neq \frac{1}{2}\right)$ is

$$
a_{0} y^{s}+b_{0} y^{1-s}+\sum_{n \neq 0}\left[a_{n} \mathbf{K}_{s}^{n}(\alpha, y)+b_{n} \mathbf{I}_{s}^{n}(\alpha, y)\right] e^{2 \pi i n x} .
$$

To find solutions to (45) when $\alpha \neq 0$, make the substitution $u(y)=\operatorname{coth}(\alpha y)$ and obtain an equivalent differential equation in terms of $V=v \circ u^{-1}$ :

$$
V^{\prime \prime}(u)+\frac{2 u}{u^{2}-1} V^{\prime}(u)+\left\{\frac{-\lambda}{u^{2}-1}+\frac{\left(\frac{2 \pi n}{\alpha}\right)^{2}}{\left(u^{2}-1\right)^{2}}\right\} V(u)=0
$$

A pair of linearly independent solutions to equation (48) is given by $P_{-s}^{-\frac{2 \pi|n|}{\alpha}}(u)$ and $Q_{-s}^{\frac{2 \pi|n|}{\alpha}}(u)$ where $P_{\nu}^{\mu}$ and $Q_{\nu}^{\mu}$ are the associated Legendre functions of the 
first and second kind respectively [Er], [O]. Thus, a pair of linearly independent solutions of (44) is given by

$$
\mathbf{P}_{s}^{n}(\alpha, y)=\Gamma\left(1+2 \pi|n| \alpha^{-1}\right) P_{-s}^{-\frac{2 \pi|n|}{\alpha}}(\operatorname{coth}(\alpha y))
$$

and

$$
\mathbf{Q}_{s}^{n}(\alpha, y)=\frac{2 e^{-2 \pi^{2}|n| i \alpha^{-1}}}{\Gamma\left(2 \pi|n| \alpha^{-1}\right)} Q_{-s}^{\frac{2 \pi|n|}{\alpha}}(\operatorname{coth}(\alpha y)) .
$$

In particular, for $\alpha \neq 0$ the Fourier expansion of an eigenfunction in the cone can be written as

$$
\sum_{n=-\infty}^{\infty}\left[a_{n} \mathbf{P}_{s}^{n}(\alpha, y)+b_{n} \mathbf{Q}_{s}^{n}(\alpha, y)\right] e^{2 \pi i n x} .
$$

The behavior as $y$ tends to infinity of $\mathbf{K}_{s}^{n}(\alpha, y)$ and $\mathbf{I}_{s}^{n}(\alpha, y)$ can be deduced from [Le], (5.11.9) and (5.11.10):

$$
\begin{aligned}
\mathbf{K}_{s}^{n}(\alpha, y) & \sim e^{-2 \pi|n| y}, \\
\mathbf{I}_{s}^{n}(\alpha, y) & \sim e^{2 \pi|n| y} .
\end{aligned}
$$

The behavior as $y$ tends to infinity of $\mathbf{P}_{s}^{n}(\alpha, y)$ and $\mathbf{Q}_{s}^{n}(\alpha, y)$ can be deduced from [Er], Section 3.9.1, (3),(5), and (12):

$$
\begin{aligned}
& \mathbf{P}_{s}^{n}(\alpha, y) \sim e^{-2 \pi|n| y}, \\
& \mathbf{Q}_{s}^{0}(\alpha, y) \sim \alpha y, \\
& \mathbf{Q}_{s}^{n}(\alpha, y) \sim e^{2 \pi|n| y} \quad(n \neq 0) .
\end{aligned}
$$

A.2. Estimates on $L^{2}$ eigenfunctions in a model cone. For the model hyperbolic cone one can define the associated Laplace-Beltrami $\Delta_{\alpha}$ as in (1). Note that for $f \in L^{2}\left(\alpha^{2}(\sinh (\alpha y))^{-2} d x d y\right) \cap C^{2}, T_{L_{\alpha} f}=\Delta_{\alpha} T_{f}$ where for $\phi \in L^{2}, T_{\phi}$ denotes the distribution $T_{\phi}(\psi)=\langle\phi, \psi\rangle_{L^{2}}$. A function $f$ will be called an $L^{2}$ eigenfunction of $\Delta_{\alpha}$ in $C_{b}$ if and only if for all bounded $C^{\infty}$ functions $\psi$ with support in $\{(x, y): y>b ; 0 \leq x \leq 1\}$ one has $\left(\Delta_{\alpha}-\lambda\right) T_{f}(\psi)=0$. Note that elliptic regularity implies that $f \in C^{\infty}$. In particular, $\left(L_{\alpha}-\lambda\right) f=0$. Thus, $f$ has an expansion of the form (47) or (49). An application of Green's formula to (1) shows that in either expansion the coefficients $b_{n}$ of $f \in L^{2}$ must be zero. For otherwise one obtains some derivative of the Dirac $\delta$ at the cone point $(y=\infty)$ as the result of applying $\Delta_{\alpha}$ to $f$. Further, if $\lambda=s(1-s)$ is real, then the functions $\left|y^{s}\right|=\left|y^{1-s}\right|=y^{\frac{1}{2}}$ are not in $L^{2}\left(y^{-2} d x d y\right)$. In particular, an $L^{2}$ eigenfunction of $\Delta_{0}$ in $C_{b}$ must have trivial zeroeth Fourier coefficient for large $y$. Hence for $\alpha=0$, an $L^{2}$ eigenfunction has the expansion

$$
\sum_{n \neq 0} a_{n} \mathbf{K}_{s}^{n}(\alpha, y) e^{2 \pi i n x}
$$


For $\alpha \neq 0$, an $L^{2}$ eigenfunction has the expansion

$$
\sum_{n=-\infty}^{\infty} a_{n} \mathbf{P}_{s}^{n}(\alpha, y) e^{2 \pi i n x}
$$

The following lemma will be useful in determining the asymptotic behavior of an $L^{2}$ eigenfunction and its derivatives.

Lemma A.1. Let $\left\{a_{n}\right\}_{n=1}^{\infty} \subset \mathbf{C}$. Let $\left\{f_{n}\right\}_{n=1}^{\infty}$ be a sequence of complex-valued functions on $\mathbf{R}^{+}$. Suppose that

$$
\sum_{i=1}^{\infty}\left|a_{n}\right|^{2}\left|f_{n}(b)\right|^{2}=M<\infty
$$

and that there exist $C_{1}, C_{2}, \beta>0$, such that for each $n \in \mathbf{Z}^{+}$and each $y>b$

$$
C_{1} e^{-\beta n y} \leq\left|f_{n}(y)\right| \leq C_{2} e^{-\beta n y} .
$$

Then for all $y>\frac{\ln 2}{\beta}+b$

$$
\left|\sum_{n=1}^{\infty} a_{n} f_{n}(y)\right| \leq 2 \sqrt{M} \frac{C_{2}}{C_{1}} e^{\beta b} e^{-\beta y} .
$$

Further, if $p(n)$ is of at most polynomial growth and $\epsilon>0$, then there exists $b_{1}>0$ such that for all $y>b_{1}$

$$
\left|\sum_{n=1}^{\infty} p(n) a_{n} f_{n}(y)\right| \leq 2 \sqrt{M} \frac{C_{2}}{C_{1}} e^{\beta b} e^{-(\beta-\epsilon) y} .
$$

Proof. From estimate (58) one finds that

$$
\left(\sum_{n \neq 0}\left|a_{n}\right|^{2} e^{-2 \beta n b}\right)^{\frac{1}{2}} \leq \frac{1}{C_{2}} \sqrt{M}
$$

and that

$$
\sum_{n \neq 0}\left|a_{n} f_{n}(y)\right| \leq 2 \sum_{n \neq 0}\left|a_{n}\right| e^{-\beta n b} e^{-\beta n(y-b)} .
$$

Application of the Cauchy-Schwarz inequality for $l^{2}$ gives

$$
\sum_{n \neq 0}\left|a_{n} f_{n}(y)\right| \leq C_{1}\left(\sum_{n \neq 0}\left|a_{n}\right|^{2} e^{-2 \beta n b}\right)^{\frac{1}{2}}\left(\sum_{n \neq 0} e^{-2 \beta n(y-b)}\right)^{\frac{1}{2}} .
$$

Summing the geometric series and applying estimate (61) one finds

$$
\sum_{n \neq 0}\left|a_{n} f_{n}(y)\right| \leq \sqrt{M} \frac{C_{1}}{C_{2}}\left(\sum_{n \neq 0}\left|a_{n}\right|^{2} e^{-2 \beta n b}\right)^{\frac{1}{2}}\left(\frac{2 e^{-2 \beta(y-b)}}{1-e^{-2 \beta(y-b)}}\right)^{\frac{1}{2}} .
$$


It follows that for $y>\frac{\ln 2}{\beta}+b$ one has estimate (59). Estimate (60) follows from essentially the same argument. In particular, one finds that

$$
\left|\sum_{n \neq 0} a_{n} f_{n}(y)\right| \leq C_{1}\left(\sum_{n \neq 0}\left|a_{n}\right|^{2} e^{-2 \beta n b}\right)^{\frac{1}{2}}\left(\sum_{n \neq 0} p(n) e^{-2 \beta n(y-b)}\right)^{\frac{1}{2}} .
$$

But since $p$ has at most polynomial growth, for each $\epsilon>0$ there exists $b_{1}$ such that for all $y>b_{1}$

$$
\sum_{n \neq 0} p(n) e^{-2 \beta n(y-b)}<\sum_{n \neq 0} e^{-2(\beta-\epsilon) n(y-b)}
$$

The proof of (60) concludes as does the proof of (59).

Lemma A.2. Let $f$ be an $L^{2}$ eigenfunction of $\Delta_{\alpha}$ in $C_{b}$ with eigenvalue $\lambda$. Let $b>\lambda(2 \pi)^{-1}$. If $\alpha \neq 0$, then further suppose that $f$ has null zeroeth Fourier coefficient for $y>b$. For all $y>\frac{\ln 2}{2 \pi}+b$ one has

$$
|f(x, y)| \leq 16 e^{2 \pi b}\left(\int_{0}^{1}|f(s, b)|^{2} d s\right)^{\frac{1}{2}} e^{-2 \pi y}
$$

Proof. Apply Lemma A.1 to the expansions (55) and (56). Parseval's formula gives estimate (57). The estimate (58) follows from the next lemma, Lemma A.3.

Lemma A.3. For $y>\frac{\lambda}{2 \pi|n|}$,

$$
\frac{1}{4} e^{-2 \pi|n| y} \leq\left|\mathbf{P}_{s}^{n}(\alpha, y)\right| \leq 2 e^{-2 \pi|n| y}
$$

and

$$
\frac{1}{4} e^{-2 \pi|n| y} \leq\left|\mathbf{K}_{s}^{n}(y)\right| \leq 2 e^{-2 \pi|n| y}
$$

Proof. The proof is an application of the Liouville-Green Approximation Method $^{4}$ to equation (48). For the sake of completeness, a statement of the main theorem on the Liouville-Green Approximation ([O], p.203) is provided below. In it $\mathscr{V}_{x, x^{\prime}}$ refers to the total variation of a function on the interval having endpoints $x$ and $x^{\prime}$.

Theorem A.1 (The Liouville-Green Approximation). Suppose that $f$ is a positive $C^{2}$ function on $\left(a_{+}, a_{-}\right)$and that $g$ is a continuous function on $\left(a_{+}, a_{-}\right)$.

\footnotetext{
${ }^{4}$ Otherwise known as WKB. See [O] for a discussion concerning attribution.
} 
Then the differential equation

$$
w^{\prime \prime}=(g(x)+f(x)) w
$$

has two solutions given by

$$
w_{ \pm}=f^{-\frac{1}{4}}(x) \exp \left[ \pm \int f^{\frac{1}{2}}(x) d x\right]\left(1+\epsilon_{ \pm}(x)\right)
$$

where

$$
\left|\epsilon_{ \pm}(x)\right| \leq e^{\frac{1}{2} \mathscr{V}_{a_{ \pm}, x}(F)}-1
$$

where

$$
F(x)=\int\left(f^{-\frac{1}{4}} \frac{d^{2}}{d x^{2}}\left(f^{-\frac{1}{4}}\right)-g f^{-\frac{1}{2}}\right) d x .
$$

To apply this theorem in the present proof, we make the substitution $W(u)=$ $\left(u^{2}-1\right)^{-\frac{1}{2}} V(u)$ into equation (48). We obtain an equivalent differential equation to which the theorem applies:

$$
W^{\prime \prime}(u)=\left(\frac{\lambda}{u^{2}-1}-\frac{1}{\left(u^{2}-1\right)^{2}}+\frac{(2 \pi|n| / \alpha)^{2}}{\left(u^{2}-1\right)^{2}}\right) W(u) .
$$

Let $f(u)=(2 \pi n)^{2}\left(\alpha\left(u^{2}-1\right)\right)^{-2}$ and $g(u)=\lambda(t)\left(u^{2}-1\right)^{-1}-\left(u^{2}-1\right)^{-2}$. To study the asymptotic behavior at the cone point we set $a_{+}=1$ and let $a_{-}=u_{0}$ where $u_{0}>1$.

After some computation one finds that

$$
W_{ \pm}=\left(u^{2}-1\right)^{\frac{1}{2}}\left(\frac{u-1}{u+1}\right)^{\frac{ \pm 2 \pi n}{2 \alpha}}\left[1+\epsilon_{ \pm}(\alpha, u)\right]
$$

One also finds that $F(u)=-\lambda u$. Thus, $\mathscr{V}_{1, u}(F)=\lambda(u-1)$ and $\mathscr{V}_{u_{0}, u}(F)=$ $\lambda\left(u_{0}-u\right)$. Therefore,

$$
\begin{aligned}
& \left|\epsilon_{+}(n, \alpha, u)\right| \leq \exp \left\{\frac{\alpha|\lambda|(u-1)}{4 \pi n}\right\}-1, \\
& \left|\epsilon_{-}(n, \alpha, u)\right| \leq \exp \left\{\frac{\alpha|\lambda|\left(u_{0}-u\right)}{4 \pi n}\right\}-1 .
\end{aligned}
$$

Upon making the reverse substitutions $V(u)=\left(u^{2}-1\right)^{\frac{1}{2}} W(u)$ and $u=$ $\operatorname{coth}(\alpha y)$, one obtains

$$
V_{ \pm}(x)=e^{\mp 2 \pi|n| y}\left[1+\epsilon_{ \pm}(\alpha, u)\right]
$$

where

$$
\begin{aligned}
& \left|\epsilon_{+}(n, \alpha, u)\right| \leq \exp \left\{\frac{\alpha|\lambda|(\operatorname{coth}(\alpha y)-1)}{4 \pi|n|}\right\}-1 \\
& \left|\epsilon_{-}(n, \alpha, u)\right| \leq \exp \left\{\frac{\alpha|\lambda|\left(y_{0}-\operatorname{coth}(\alpha y)\right)}{4 \pi|n|}\right\}-1
\end{aligned}
$$


with $y_{0}=\operatorname{coth}^{-1}\left(\alpha y_{0}\right)$. From (52) it follows that $V_{+}=\mathbf{P}_{s}^{n}(\alpha, y)$. Since $\alpha(\operatorname{coth}(\alpha y)-1) \leq y^{-1}$, one has

$$
e^{-2 \pi|n| y}\left[2-\exp \left(\frac{\lambda}{4 \pi|n| y}\right)\right] \leq\left|\mathbf{P}_{s}^{n}(\alpha, y)\right| \leq e^{-2 \pi|n| y}\left[\exp \left(\frac{\lambda}{4 \pi|n| y}\right)\right]
$$

Estimate (63) follows from (66). Note that equation (45) is a differential equation whose coefficients depend analytically on $\alpha$ for all $\alpha$. The elementary theory of ordinary differential equations assures the continuity of solutions, and hence estimate (64) follows from (63) and the normalized asymptotics (50) and (52).

Lemma A.4. Let $f$ be an $L^{2}$ eigenfunction of $\Delta_{\alpha}$ in $C_{b}$ with eigenvalue $\lambda$. If $\alpha \neq 0$, then further suppose that $f$ has null zeroeth Fourier coefficient for $y>b$. Then for each $i$ and $j \in \mathbf{Z}^{+}$and $\epsilon>0$, there exist constants $b^{\prime}$ and $c$ such that for all $y>b^{\prime}$

$$
\left|\frac{\partial^{i}}{\partial x^{i}} \frac{\partial^{j}}{\partial y^{j}} f(x, y)\right| \leq c^{\frac{i+j}{2}} e^{-2 \pi y}
$$

Proof. Note that $f$ satisfies

$$
\left(\Delta_{E u c}-\lambda \alpha^{-2}(\sinh (\alpha y))^{-2}\right) f(x, y)=0
$$

on each square $S_{m}=\{(x, y): m \leq y \leq m+1 ; 0 \leq x \leq 1\}$. Thus, Schauder estimates provide a $C$ such that

$$
|f|_{C^{2, \alpha}\left(S_{m}\right)} \leq|f|_{C^{0}}
$$

Since the constant $C$ depends only on the shape of the domain, the ellipticity constant of the operator, and the bounds on the coefficients, one can choose $C$ to be uniform in $m$. By applying Lemma A.2, one obtains on the entire strip $I=\left\{(x, y): y>b^{*} ; 0 \leq x \leq 1\right\}$

$$
|f|_{C^{2, \alpha}(I)} \leq C^{\prime} e^{-2 \pi y}
$$

for constants $C^{\prime}$ and $b^{*}$. Note that

$$
\Delta_{E u c} f(x, y)=\lambda \alpha^{-2}(\sinh (\alpha y))^{-2} f(x, y) \text {. }
$$

Hence another application of the Schauder estimates implies that

$$
\left|\frac{\partial^{i}}{\partial x^{i}} \frac{\partial^{j}}{\partial y^{j}} f(x, y)\right|_{C^{2, \alpha}(I)} \leq C_{1} \lambda\left|\frac{\partial^{i}}{\partial x^{i}} \frac{\partial^{j}}{\partial y^{j}} f(x, y)\right|_{C^{\alpha}(I)} .
$$

Iteration of this estimate yields the result.

To determine the asymptotics of the first variation of an eigenfunction branch, the following two lemmas are provided, Lemma A.6 and Lemma A.7. The first concerns the case $\alpha>0$; the second concerns the case $\alpha=0$ in which the cone is a cusp. The proofs are entirely different for the following reasons: 
For the case $\alpha>0$, one the zeroeth Fourier coefficient is a multiple of a single function $\mathbf{P}_{S}^{0}$ for which there are explicit integral expansions which can be differentiated with respect to $\alpha$ for $\alpha>0$ (Lemma A.5). The differentiation of such an expression breaks down at $\alpha=0$. Instead another more traditional approach is used. In particular, one integrates the variation of the eigenbranch against the resolvent kernel. This approach works for $\alpha=0$ because we have that the first variation belongs to $L^{2}$ (Lemma 4.1). Note that it is not a priori known whether the first variation belongs to $L^{2}$ if $\alpha>0$.

Lemma A.5. Let $\alpha>0$. There exist functions $f_{n}(\alpha, y)$ and constants $c_{n}$, $C_{1}(\alpha), C_{2}(\alpha)$, and $b(\alpha)$ such that:

(1) $c_{n}(\alpha)$ has at most polynomial growth in $n$,

(2)

$$
\frac{d}{d \alpha} \mathbf{P}_{s(\alpha)}^{n}(\alpha, y)=c_{n}(\alpha) f_{n}(\alpha, y)
$$

and

(3) for $y>b(\alpha)$

$$
C_{1}(\alpha) e^{-2|n| \pi y} \leq\left|f_{n}(\alpha, y)\right| \leq C_{2}(\alpha) e^{-2 \pi|n| y}
$$

Proof. The following integral representation for $P_{\nu}^{\mu}$ is given in [Er], Section 3.7, (6):

$$
P_{\nu}^{\mu}(z)=\frac{2^{\mu}\left(z^{2}-1\right)^{-\frac{\mu}{2}}}{\pi^{\frac{1}{2}} \Gamma\left(\frac{1}{2}-\mu\right)} \int_{0}^{\pi}\left[z+\left(z^{2}-1\right)^{\frac{1}{2}} \cos (t)\right]^{\nu+\mu}(\sin (t))^{-2 \mu} d t .
$$

By substituting $\mu=\frac{-2 \pi n}{\alpha}, z=\operatorname{coth}(\alpha y)$, and $\nu=-s(\alpha)$ one obtains

$$
\begin{aligned}
\mathbf{P}_{s(\alpha)}^{n}(\alpha, y)= & \frac{\Gamma\left(1+2 \pi|n| \alpha^{-1}\right)}{\pi^{\frac{1}{2}} \Gamma\left(\frac{1}{2}+2 \pi|n| \alpha^{-1}\right)}(\sinh (\alpha y))^{-\frac{2 \pi|n|}{\alpha}} \\
& \times \int_{0}^{\pi}\left(\frac{\sinh (\alpha y)}{\cosh (\alpha y)-\cos (t)}\right)^{s(\alpha)+2 \pi|n| \alpha^{-1}}(\sin (t))^{4 \pi|n| \alpha^{-1}} d t .
\end{aligned}
$$

By differentiating with respect to $\alpha$ one obtains a mess:

$$
\frac{d}{d \alpha} \mathbf{P}_{s(\alpha)}^{n}(\alpha, y)=A \cdot B(y) \int_{0}^{\pi}\left(E_{1}(t, y)+E_{2}(t, y)\right) \ln (\sin (t))(\sin (t))^{4 \pi n \alpha^{-1}} d t
$$

where

$$
A=\frac{2 \pi|n|}{\alpha^{2}} \frac{\Gamma\left(1+\frac{2 \pi|n|}{\alpha}\right)}{\pi^{\frac{1}{2}} \Gamma\left(\frac{1}{2}+\frac{2 \pi|n|}{\alpha}\right)}\left(\psi\left(2^{-1}+2 \pi|n| \alpha^{-1}\right)-\psi\left(1+2 \pi|n| \alpha^{-1}\right)\right)
$$

$$
B(y)=\frac{2 \pi|n|}{\alpha^{2}}(\sinh (\alpha y))^{-1-\frac{2 \pi|n|}{\alpha}}(\ln (\sinh (\alpha y)) \sinh (\alpha y)-\alpha y \cosh (\alpha y)),
$$


(71)

$$
E_{1}(t, y)=\frac{y(1-\cos (t) \cosh (\alpha y))\left(\frac{2 \pi|n|}{\alpha}+s(\alpha)\right)(\sinh (\alpha y))^{-1+s(\alpha)+\frac{2 \pi|n|}{\alpha}}}{(\cosh (\alpha y)-\cos (t))^{1+s(\alpha)+\frac{2 \pi|n|}{\alpha}}},
$$

and

$$
\begin{aligned}
E_{2}(t, y)= & \ln \left(\frac{\sinh (\alpha y)}{\cosh (\alpha y)-\cos (t)}\right)\left(\frac{\sinh (\alpha y)}{\cosh (\alpha y)-\cos (t)}\right)^{-1+s(\alpha)+2 \pi|n| \alpha^{-1}} \\
& \times\left(-2 \pi|n| \alpha^{-1}+s^{\prime}(\alpha)\right)
\end{aligned}
$$

where $\psi$ is the logarithmic derivative of $\Gamma . \psi$ has at most logarithmic growth in $n$ [Er], Section 1.18, (7). Thus, from [Er], Section 1.18, (4), one sees that $A$ has at most polynomial growth in $n$. Note that as $y$ tends to infinity

$$
B(y) \sim \frac{2 \pi n}{\alpha^{2}} e^{-2 \pi|n|} .
$$

Also note that $E_{1}(t, y)$ and $E_{2}(t, y)$ tend to zero uniformly in $t$ as $y$ tends to infinity, and that the growth in $n$ is at most polynomial. Putting all these things together, one has the result.

Lemma A.6. Let $\psi_{\alpha}$ be a family of $L^{2}$ eigenfunctions of $\Delta_{\alpha}$ in $C_{b}$ all of which have null zeroeth Fourier coefficient for $y>b$. Let $\lambda_{\alpha}=s(\alpha)(1-s(\alpha))$ denote the real eigenvalue of $\psi_{\alpha}$. Suppose that the family $\psi_{\alpha}$ varies real-analytically in $\alpha$ with respect to sup norm on each compact set in the model cone. Let $f$ be an $L^{2}$ eigenfunction of $\Delta_{\alpha_{0}}$ for $\alpha_{0} \neq 0$ and let $\dot{\psi}$ denote $\left.\frac{d}{d \alpha} \psi\right|_{\alpha=\alpha_{0}}$. Then

$$
\lim _{y \rightarrow 0} \int_{y=a} \frac{\partial}{\partial y}(\dot{\psi}(x, y)) f(x, y) d x=0
$$

and

$$
\lim _{y \rightarrow 0} \int_{y=a} \dot{\psi}(x, y) \frac{\partial}{\partial y}(f(x, y)) d x=0 .
$$

Proof. The functions $\psi_{\alpha}$ have Fourier expansions given by (56)

$$
\psi_{\alpha}(x, y)=\sum_{n=-\infty}^{\infty} a_{n}(\alpha) \mathbf{P}_{s}^{n}(\alpha, y) e^{2 \pi i n x} .
$$

Writing $\dot{\mathbf{P}}_{s}^{n}$ for $\left.\frac{d}{d \alpha}\right|_{\alpha=\alpha_{0}} \mathbf{P}_{s}^{n}(\alpha, y)$, one has

$$
\dot{\psi}(x, y)=\sum_{n=-\infty}^{\infty}\left[a_{n}^{\prime}(\alpha) \mathbf{P}_{s}^{n}(\alpha, y)+a_{n}(\alpha) \dot{\mathbf{P}}_{s}^{n}(\alpha, y)\right] e^{2 \pi i n x} .
$$

$f$ also has a Fourier expansion of the form (56)

$$
\sum_{n=-\infty}^{\infty} b_{n}(\alpha) \mathbf{P}_{s}^{n}(\alpha, y) e^{2 \pi i n x}
$$


One has

$$
\begin{aligned}
\int_{y=a} & \frac{\partial}{\partial y}(\dot{\psi}(x, y)) f(x, y) d x \\
= & \sum_{n=-\infty}^{\infty}\left[a_{n}^{\prime}(\alpha) \partial_{y} \mathbf{P}_{s}^{n}(\alpha, y)+a_{n}(\alpha) \partial_{y} \dot{\mathbf{P}}_{s}^{n}(\alpha, y)\right] b_{-n}(\alpha) \mathbf{P}_{s}^{-n}(\alpha, y) \\
\leq & \left(\sum_{n=-\infty}^{\infty}\left|a_{n}^{\prime}(\alpha)\right|^{2} \partial_{y}\left|\mathbf{P}_{s}^{n}(\alpha, y)\right|^{2}\right)^{\frac{1}{2}} \\
& \left.+\left(\sum_{n=-\infty}^{\infty}\left|a_{n}(\alpha)\right|^{2} \partial_{y}\left|\dot{\mathbf{P}}_{s}^{n}(\alpha, y)\right|^{2}\right)^{\frac{1}{2}}\right) \\
& +\left(\sum_{n=-\infty}^{\infty}\left|b_{n}(\alpha)\right|^{2}\left|\mathbf{P}_{s}^{n}(\alpha, y)\right|^{2}\right)^{\frac{1}{2}}
\end{aligned}
$$

Note that since $f$ is an $L^{2}$ eigenfunction, $f$ is bounded. Therefore (73) would follow from

$$
\lim _{y \rightarrow \infty} \sum_{n=-\infty}^{\infty}\left|a_{n}^{\prime}(\alpha)\right|^{2}\left|\partial_{y} \mathbf{P}_{s}^{n}(\alpha, y)\right|^{2}=0
$$

and

$$
\lim _{y \rightarrow \infty} \sum_{n=-\infty}^{\infty}\left|a_{n}(\alpha)\right|^{2}\left|\partial_{y} \dot{\mathbf{P}}_{s}^{n}(\alpha, y)\right|^{2}=0
$$

To obtain (79) and (80), Lemma A.1 is applied to the functions $\sum_{n \neq 0} a_{n}^{\prime}(\alpha) \partial_{y} \mathbf{P}_{s}^{n}(\alpha, y)$ and $\sum_{n \neq 0} a_{n}(\alpha) \partial_{y} \dot{\mathbf{P}}_{s}^{n}(\alpha, y)$, and the zeroeth Fourier coefficients are shown to tend to zero as $y$ tends to infinity. To obtain the estimate (58) one evaluates $\partial_{y} \mathbf{P}_{s}^{n}(\alpha, y)$ and $\partial_{y} \dot{\mathbf{P}}_{s}^{n}(\alpha, y)$. To this end the relation [Er], Section $3.8,(10)$, is used:

$$
\left(z^{2}-1\right) \frac{d}{d z} P_{\nu}^{\mu}(z)=(\nu-\mu+1) P_{\nu+1}^{\mu}(z)-(\nu+1) z P^{\mu}(z)
$$

By substituting $\mu=-\alpha^{-1} 2 \pi|n|, \nu=-s(\alpha)$, and $z=\operatorname{coth}(\alpha y)$, one finds that

$$
\frac{d}{d y} \mathbf{P}_{s}^{n}(\alpha, y)=\alpha(1-s) \operatorname{coth}(\alpha y) \mathbf{P}_{s}^{n}(\alpha, y)-(\alpha(1-s)+2 \pi|n|) \mathbf{P}_{1-s}^{n}(\alpha, y)
$$


and hence

$$
\begin{aligned}
\frac{d}{d y} \dot{\mathbf{P}}_{s}^{n}(\alpha, y)= & (1-s)\left[\operatorname{coth}(\alpha y)-\alpha y \sinh ^{-2}(\alpha y)\right] \mathbf{P}_{s}^{n}(\alpha, y) \\
& +\alpha(1-s) \operatorname{coth}(\alpha y) \dot{\mathbf{P}}_{s}^{n}(\alpha, y)+(s-1) \mathbf{P}_{s}^{n}(\alpha, y) \\
& -(\alpha(1-s)+2 \pi|n|) \dot{\mathbf{P}}_{s}^{n}(\alpha, y) .
\end{aligned}
$$

Using the estimate given by Lemma A.5 one obtains estimate (58). The constants absorbed into the the series $a_{n}$ are of at most polynomial growth. Thus, Lemma A.1 applies and implies that

$$
\lim _{y \rightarrow \infty} \sum_{n=\neq 0}\left|a_{n}^{\prime}(\alpha)\right|^{2}\left|\partial_{y} \mathbf{P}_{s}^{n}(\alpha, y)\right|^{2}=0
$$

and

$$
\lim _{y \rightarrow \infty} \sum_{n \neq 0}\left|a_{n}(\alpha)\right|^{2}\left|\partial_{y} \dot{\mathbf{P}}_{s}^{n}(\alpha, y)\right|^{2}=0
$$

By use of [Er], Section 3.2 (14), and [Le], (9.2.2), one finds that

$$
\frac{d}{d y} \mathbf{P}_{s}^{0}(\alpha, y)=\frac{\alpha s(1-s) F\left(s+1,2-s ; 2 ; 2^{-1}(1-\operatorname{coth}(\alpha y))\right)}{2 \sinh ^{2}(\alpha y)}
$$

and

$$
\begin{aligned}
\frac{d}{d y} \dot{\mathbf{P}}_{s}^{0}(\alpha, y)= & \frac{-\alpha y s(1-s) \operatorname{coth}(\alpha y) F\left(s+1,2-s ; 2 ; \frac{1}{2}(1-\operatorname{coth}(\alpha y))\right)}{\left.\sinh ^{2}(\alpha y)\right)} \\
& +\frac{-\alpha y s(1-s)(s+1)(2-s) F\left(s+2,3-s ; 3 ; \frac{1}{2}(1-\operatorname{coth}(\alpha y))\right)}{4 \sinh ^{4}(\alpha y)}
\end{aligned}
$$

where $F$ is the hypergeometric function. In particular, the zeroeth Fourier coefficient is $O\left(e^{-2 \alpha y}\right)$. In sum, (73) has been verified. Verification of (74) is similarly obtained.

For $\alpha=\alpha_{0}$ one has the following

Lemma A.7. Let $\psi$ be an $L^{2}$ eigenfunction of $\Delta_{0}$ in $C_{b}$ with real eigenvalue $\frac{1}{4}+r^{2}$. Suppose that $f \in L^{2}\left(y^{-2} d x d y\right)$ has trivial zeroeth Fourier coefficient for $y>b$ and satisfies

$$
\left(\Delta_{0}+\lambda\right) f=\eta \psi
$$

for some constants $\lambda=s(1-s)$ and $\eta$. Then $f$ is $O\left(e^{-2 \pi y}\right)$ as $y$ tends to infinity. 
Proof. The eigenfunction $\psi$ has the expansion (55). Elliptic regularity implies that $f$ has a Fourier expansion

$$
f=\sum_{n \neq 0} a_{n}(y) e^{2 \pi i n x} .
$$

Equation (85) implies that

$$
y^{2}\left(\partial_{y}^{2}-k^{2}+\lambda\right) a_{n}(y)=\left(y^{2}+\eta\right) c_{n} \mathbf{K}_{i r}^{n}(y)
$$

for each $n \neq 0$, where $k=2 \pi n$. The Green's function $G_{n}$ for the operator $y^{2}\left(\partial_{y}^{2}-k^{2}+s(1-s)\right)$ is given by [La], p. 291,

$$
k G_{n}(y, v)= \begin{cases}\mathbf{I}_{s}^{n}(v) \mathbf{K}_{s}^{n}(y), & v<y, \\ \mathbf{I}_{s}^{n}(y) \mathbf{K}_{s}^{n}(v), & v>y\end{cases}
$$

Thus by taking the $L^{2}\left(y^{-2} d y\right)$ inner product of each side of equation (86) with $G_{n}$ one obtains

$$
\begin{aligned}
a_{n}(y)= & \mathbf{K}_{s}^{n}(y) \int_{0}^{y}\left(1+\frac{\eta}{v^{2}}\right) c_{n} \mathbf{K}_{i r}^{n}(v) \mathbf{I}_{s}^{n}(v) d v \\
& +\mathbf{I}_{s}^{n}(y) \int_{y}^{\infty}\left(1+\frac{\eta}{v^{2}}\right) c_{n} \mathbf{K}_{i r}^{n}(v) \mathbf{K}_{s}^{n}(v) d v
\end{aligned}
$$

The asymptotics (50) and (51) imply that there exists $C$ and $C^{\prime}>0$ such that

$$
\begin{gathered}
\left|\left(1+\frac{\eta}{y^{2}}\right) \mathbf{K}_{i r}^{n}(y) \mathbf{I}_{s}^{n}(y)\right| \leq C, \\
\left|\left(1+\frac{\eta}{y^{2}}\right) \mathbf{K}_{i r}^{n}(y) \mathbf{K}_{s}^{n}(y)\right| \leq C e^{-4 \pi|n| y}, \\
\left|\mathbf{I}_{i r}^{n}(y)\right| \leq C^{\prime} e^{2 \pi|n| y},
\end{gathered}
$$

and

$$
\left|\mathbf{K}_{i r}^{n}(y)\right| \leq C^{\prime} e^{-2 \pi|n| y}
$$

for $y>y_{0}$. Applying these asymptotics to (87), one obtains for $y>y_{0}$,

$$
\begin{aligned}
\left|a_{n}(y)\right| & \leq c_{n}\left(C y\left|\mathbf{K}_{s}^{n}(y)\right|+\mathbf{I}_{s}^{n}(y) C e^{-4 \pi y}\right) \\
& \leq c_{n} C C^{\prime} e^{-2 \pi y}(y+1) .
\end{aligned}
$$

The sequence $\left\{c_{n}\right\}$ belong to $l^{2}$ and thus the sum $\sum_{n \neq 0} c_{n} e^{-2 \pi n y}(y+1)$ converges. A geometric series and a Schwarz inequality argument similar to that given in Lemma A.1 shows that the function defined by this series is $O\left(e^{-2 \pi y}\right)$. The conclusion follows from the estimate $|f| \leq \sum_{n \neq 0}\left|a_{n}(y)\right|$. 
A.3. The Coincidence Principle. Here we provide the proof of Lemma 3.1. It suffices to understand the behavior of the zeroeth Fourier coefficient. By using [Le], Ex. 2, p. 200, one obtains

$$
\begin{aligned}
\mathbf{P}_{s}^{0}(\alpha, y) & =P_{-s}^{0}(\operatorname{coth}(\alpha y)) \\
& =\left(1-e^{-2 \alpha y}\right)^{s} F\left(s, s ; 1 ; e^{-2 \alpha y}\right)
\end{aligned}
$$

where $F$ denotes the hypergeometric function. By applying a connection formula [Le], (9.5.7), to equation (90), one obtains an equation useful in studying the behavior of $\mathbf{P}_{s}^{0}(\alpha, y)$ as $\alpha$ tends to zero

$$
\begin{aligned}
\mathbf{P}_{s}^{0}(\alpha, y) & =A_{s}\left(1-e^{-2 \alpha y}\right)^{s} F\left(s, s ; 2 s ; 1-e^{-2 \alpha y}\right) \\
& +B_{s}\left(1-e^{-2 \alpha y}\right)^{1-s} F\left(1-s, 1-s ; 2-2 s ; 1-e^{-2 \alpha y}\right)
\end{aligned}
$$

where $A_{s}=\Gamma(1-2 s)(\Gamma(-s))^{2}$ and $B_{s}=\Gamma(2 s-1)(\Gamma(s))^{2}$. If $s=\frac{1}{2}+i r$, then equation (91) reduces to

$$
\mathbf{P}_{s}^{0}(\alpha, y)=2 \operatorname{Re}\left(A_{s}\left(1-e^{-2 \alpha y}\right)^{s} F\left(s, s ; 2 s ; 1-e^{-2 \alpha y}\right)\right) .
$$

Using (92), we obtain a proof of the Coincidence Principle, Lemma 3.1.

Proof of the Coincidence Principle: Lemma 3.1. It suffices to show that the zeroeth Fourier coefficient of $\psi_{\alpha}$ in the perturbed cone point vanishes at $b$ for a sequence of $\alpha_{n}$ which converges to zero. Let $\lambda_{\alpha}=s_{\alpha}\left(1-s_{\alpha}\right)=\frac{1}{2}+r_{\alpha}$ be the eigenvalue branch associated to $\Delta_{\gamma}^{b}$. Note that Theorem 5.1 implies that $r_{\alpha}$ is decreasing as $\alpha$ decreases. Since $r_{\alpha} \geq 0, r_{\alpha}$ limits to, say, $r_{0}$ as $\alpha$ tends to zero.

The eigenfunctions of $\Delta_{\gamma}^{b}$ have finite values at the perturbed cone point for $\alpha>0$ and thus have zeroeth Fourier coefficient in that cone equal to some multiple of the Legendre function $P_{-s}^{0}$. Since the eigenvalues are real and at least $\frac{1}{4}$, we can apply equation (91):

$$
P_{-s}^{0}(\operatorname{coth}(\alpha b))=2 \operatorname{Re}\left(A\left(s_{\alpha}\right)\left(1-e^{-2 \alpha b}\right)^{s_{\alpha}} F\left(s_{\alpha}, s_{\alpha} ; 2 s_{\alpha} ; 1-e^{-2 \alpha b}\right)\right) .
$$

Define

$$
H(\alpha)=\sum_{n=0}^{\infty}(-1)^{n} \frac{2^{n} \alpha^{n} b^{n}}{n !} .
$$

Also set $A\left(\frac{1}{2}+i r_{\alpha}\right)=a_{\alpha} e^{i \theta_{\alpha}}$. Then by expanding $\left(1-e^{-2 \alpha b}\right)$ in $\alpha$ and expanding the hypergeometric function in its series representation, one obtains

$$
\alpha^{-\frac{1}{2}} P_{-s_{\alpha}}^{0}(\operatorname{coth}(\alpha b))=2 a_{\alpha} b^{\frac{1}{2}} \cos \left(r_{\alpha}[\log \alpha+\log b H(\alpha)]+\theta_{\alpha}\right)+O(\alpha)
$$

where $O(\alpha)$ is some function bounded in absolute value by a constant times $\alpha$. Note that $\log b H(\alpha)$ tends to $\log b$ and $\theta_{\alpha}$ tends to $\theta_{0}$ as $\alpha$ tends to zero. 
Thus it is easy to see that if $r_{\alpha}$ is bounded away from zero, then

$$
2 a_{\alpha} b^{\frac{1}{2}} \cos \left(r_{\alpha}[\log \alpha+\log (b H(\alpha))]+\theta_{\alpha}\right)
$$

oscillates infinitely often as $\alpha$ tends to zero. The amplitude converges to $2 a_{0} b^{\frac{1}{2}}>0$, and thus since the error term is $O(\alpha)$, a multiple of the zeroeth Fourier coefficient, $\alpha_{n}^{-\frac{1}{2}} P_{-s_{\alpha_{n}}}^{0}\left(\operatorname{coth}\left(\alpha_{n} b\right)\right)$, must vanish for a sequence $\left\{\alpha_{n}\right\}$ tending to zero.

\section{ACKNOWLEDGMENT}

The results contained herein and in $[\mathrm{J}]$ constitute the author's doctoral dissertation at the University of Maryland. The author takes great pleasure in thanking his thesis advisor Scott Wolpert for his support, both moral and technical. The author also thanks Peter Sarnak for his encouragement and very constructive criticism, and Isaac Efrat for his encouragement and helpful comments. Lastly, the author thanks the Institute for Advanced Study for its hospitality during the latter stages of manuscript preparation.

\section{REFERENCES}

[B] M. S. Berger, Nonlinearity and functional analysis, Academic Press, New York, 1977.

[BJS] L. Bers, F. John, and M. Shechter, Partial differential equations, Amer. Math. Soc., Providence, RI, 1964.

[CdV] Y. Colin de Verdiere, Pseudo-Laplacians II, Ann. Inst. Fourier 33 (1983), 87-113.

[CH] R. Courant and D. Hilbert, Methods of mathematical physics, Vol. I, Wiley, New York, 1989.

[DIPS] J.M. Deshouillers, H. Iwaniec, R.S. Phillips, and P. Sarnak, Maass cusp forms, Proc. Nat. Acad. Sci. U.S.A. 82 (1985), 3533-3534.

[Ef] I. Efrat, On the discrete spectrum of certain discrete groups, Bull. Amer. Math. Soc. (N.S.) 24 (1991), 125-130.

[Er] A. Erdelyi (ed.), Higher transcendental functions: The Bateman manuscript project, Vol. 1, McGraw-Hill, New York, 1953.

[G] S. Gelbart, Automorphic forms on adele groups, Princeton Univ. Press, Princeton, NJ, 1975.

[GGP] I. M. Gelfand, M. I. Graev, and I. I. Pyatetskii-Shapiro, Representation theory and automorphic functions, Academic Press, New York, 1990.

[GB] L. Greenberg and I. Babuška, A continuous analogue of Sturm sequences in the context of Sturm-Liouville equations, Siam. J. Numer. Anal. 26 (1989), 920-945.

[H1] D. Hejhal, The Selberg trace formula for $\operatorname{PSL}(2, \mathbf{R})$, Vol. II, Lecture Notes in Math., vol. 1001, Springer-Verlag, Berlin and New York, 1983.

[H2] _ Eigenvalues of the Laplacian for Hecke triangle groups, Mem. Amer. Math. Soc. 97 (1992), Number 469.

[Ji] L. Ji, Degeneration of the pseudo-Laplace operator for hyperbolic Riemann surfaces, J. Differential Geometry 38 (1993), 263-313.

[J] C. Judge, The variation of constant curvature surfaces with conical singularities, Preprint, 1993.

[JZ] L. Ji and S. Zelditch, Hyperbolic cusp forms and spectral simplicity on compact hyperbolic surfaces, Preprint, 1993.

[K] T. Kato, Perturbation theory for linear operators, Springer-Verlag, Berlin, 1980.

[La] S. Lang, $S L(2, \mathbf{R})$, Springer-Verlag, New York, 1974. 
[Le] N. N. Lebedev, Special functions and their applications, Dover, New York, 1972.

[LP] P. Lax and R. Phillips, Scattering theory for automorphic functions, Princeton Univ. Press, Princeton, NJ, 1976.

[Lu] W. Luo, On the nonvanishing of Rankin-Selberg L-functions, Duke Math. J. 69 (1993), 411-427.

[O] F.W.J. Olver, Asymptotics and special functions, Academic Press, New York, 1974.

[Pe] I. M. Petridis, Scattering theory for automorphic functions and its relation to L-functions, Thesis, Stanford, 1992.

[PS1] R.S. Phillips and P. Sarnak, On cusp forms for cofinite subgroups of $\operatorname{PSL}(2, \mathbf{R})$, Invent. Math. 80 (1985), 339-364.

[PS2] On Weyl's Law for noncompact finite volume surfaces, Comm. Pure. Appl. Math. 38 (1985).

[PS3] — Cusp forms for character varieties, Preprint, 1992.

[PS4] _ Automorphic spectrum and Fermi's golden rule, Preprint, 1992.

[RN] F. Riesz and B. Sz.-Nagy, Functional analysis, Dover, New York, 1990.

[Sa1] P. Sarnak, Prime geodesic theorems, Thesis, Stanford, 1980.

[Sa2] On cusp forms, Contemp. Math., vol. 53, Amer. Math. Soc., Providence, RI, 1986, pp. 393-407.

[Se1] A. Selberg, Göttingen lectures, 1954.

[Se2] _ Harmonic analysis and discontinuous groups in weakly symmetric Riemannian spaces with applications to Dirichlet series, J. Indian Math. Soc. 20 (1956), 47-87.

[T] A. Terras, Harmonic analysis on symmetric spaces, Vol. I, Springer-Verlag, Berlin and New York, 1985.

[U] K. Uhlenbeck, Generic properties of eigenfunctions, Amer. J. Math. 98 (1976), 1059-1078.

[Wk] A. Winkler, Cusp forms and Hecke groups, J. Reine Angew. Math. 386 (1988), 187-204.

[Wp1] S. Wolpert, Spectral limits for hyperbolic surfaces I, Invent. Math. 108 (1992), 67-89.

[Wp2] _ Disappearance of cusp forms in special families, Ann. of Math. (to appear).

[V] A. B. Venkov, The spectral theory of automorphic functions, Klüwer, Dordrecht, 1990.

ABSTRACT. The perturbation theory of the Laplace spectrum of hyperbolic surfaces with conical singularities belonging to a fixed conformal class is developed. As an application, it is shown that the generic such surface with cusps has no Maass cusp forms ( $L^{2}$ eigenfunctions) under specific eigenvalue multiplicity assumptions. It is also shown that eigenvalues depend monotonically on the cone angles. From this, one obtains Neumann eigenvalue monotonicity for geodesic triangles in $\mathbf{H}^{2}$ and a lower bound of $\frac{1}{2} \pi^{2}$ for the eigenvalues of 'odd' Maass cusp forms associated to Hecke triangle groups.

Institute for Advanced Study, Princeton, New Jersey 08540

Current address: Department of Mathematics, University of Southern California, Los Angeles, California 90089

E-mail address: cjudge@math.usc.edu 